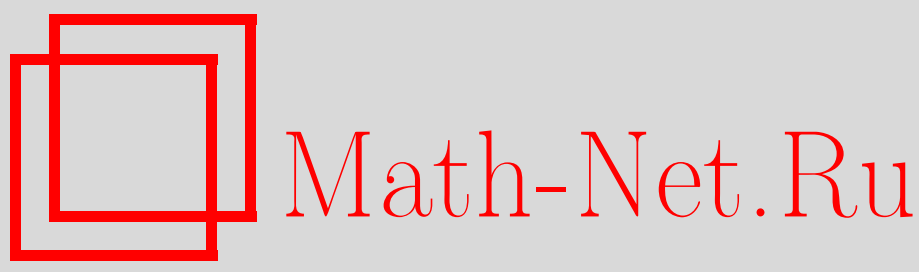

В. А. Смирнов, Алгебры Ли над операдами и их применение в теории гомотопий, Изв. РАН. Сер. матем., 1998, том 62, выпуск 3, 121-154

DOI: https://doi.org/10.4213/im198

Использование Общероссийского математического портала Math-Net.Ru подразумевает, что вы прочитали и согласны с пользовательским соглашением http: //www. mathnet.ru/rus/agreement

Параметры загрузки:

IP: 52.23 .180 .231

26 апреля 2023 г., 13:54:59 
УДК 513.83

\author{
В. А. Смирнов
}

\title{
Алгебры Ли над операдами и их применение в теории гомотопий
}

\begin{abstract}
Настоящая работа посвящена развитию алгебраического аппарата, необходимого для описания гомотопических групп топологических пространств. Как показано в заметке [1], для этого нужно использовать понятие алгебры Ли над операдой $E_{\infty}$. Здесь мы подробнее рассмотрим это понятие, докажем важнейшие его свойства и выясним вопрос об алгебраической структуре на гомотопических группах топологических пространств. В частности, покажем, что на гомотопических группах топологического пространства имеется структура $E_{\infty}$-алгебры Ли, которая в односвязном случае определяет гомотопический тип этого пространства.

Поскольку понятие операды является аналогом понятия алгебры, то вначале мы напоминаем понятия алгебры, коалгебры и рассматриваем основные конструкции над ними, которые затем переносятся на случай операд и используются при исследовании структуры алгебры и коалгебры Ли над операдой.

Библиографияя: 17 наименований.
\end{abstract}

\section{§ 1. Алгебры и коалгебры}

Пусть $A$ - дифференциальная градуированная алгебра с единицей и ассоциативным умножением $\pi: A \otimes A \rightarrow A, K$ - дифференциальная градуированная коалгебра с коединищей и ассоциативным коумножением $\nabla: K \rightarrow K \otimes K$.

Дифференциальные градуированные алгебры и коалгебры в дальнейшем мы будем называть просто алгебрами и коалгебрами соответственно.

Скрещивающей кощепью $\phi: K \rightarrow A$ называется [2] гомоморфизм, понижающий размерность на единицу и удовлетворяюший условию $d(\phi)+\phi \cup \phi=0$, где $\phi \cup \phi=\pi(\phi \otimes \phi) \nabla: K \rightarrow A$.

Для алгебры $A$ рассмотрим $B$-конструкцию $B A$, являющуюся коалгеброй, и скрешиваюшую коцепь $\beta: B A \rightarrow A$. Как градуированная коалгебра $B A$ совпадает с тензорной коалгеброй $T \Sigma A$ над надстройкой $\Sigma A$ над $A$.

Образуюшие в $B A$ обозначаются через $\left[x_{1}, \ldots, x_{n}\right], x_{i} \in A$, и имеют размерность, равную сумме размерностей $x_{i}$ плюс $n$.

Коумножение $\nabla: B A \rightarrow B A \otimes B A$ задается формулой

$$
\nabla\left[x_{1}, \ldots, x_{n}\right]=\sum_{i=1}^{n-1}\left[x_{1}, \ldots, x_{i}\right] \otimes\left[x_{i+1}, \ldots, x_{n}\right]
$$

Работа выполнена при финансовой поддержке Российского фонда фундаментальных исследований (грант № 96-01-01049).

(C) В.А. Смирнов 1998 
Скрешивающая коцепь $\beta: B A \rightarrow A$ определена формулой

$$
\beta\left[x_{1}, \ldots, x_{n}\right]= \begin{cases}x_{1}, & n=1 \\ 0, & n>1 .\end{cases}
$$

Дифференциал в коалгебре $B A$ определяется условием скрещивающей коцепи $\beta$ и имеет вид

$$
\begin{aligned}
d\left[x_{1}, \ldots, x_{n}\right]= & \sum_{i=1}^{n}(-1)^{\epsilon_{i-1}+1}\left[x_{1}, \ldots, d\left(x_{i}\right), \ldots, x_{n}\right] \\
& +\sum_{i=1}^{n-1}(-1)^{\epsilon_{i}}\left[x_{1}, \ldots, \pi\left(x_{i} \otimes x_{i+1}\right), \ldots, x_{n}\right],
\end{aligned}
$$

где $\epsilon_{i}=\operatorname{dim}\left(x_{1}\right)+\cdots+\operatorname{dim}\left(x_{i}\right)+i$.

Двойственным образом для коалгебры $K$ рассмотрим ко- $B$-конструкцию $F K$, являющуюся алгеброй, и скрещивающую коцепь $\alpha: K \rightarrow F K$. Как градуированная алгебра $F K$ совпадает с тензорной алгеброй $T \Sigma^{-1} K$ над денадстройкой $\Sigma^{-1} K$ над $K$.

Образуюшие в $F K$ обозначаются через $\left[x_{1}, \ldots, x_{n}\right], x_{i} \in K$, и имеют размерность, равную сумме размерностей $x_{i}$ минус $n$.

Умножение $\pi: F K \otimes F K \rightarrow F K$ задается формулой

$$
\pi\left(\left[x_{1}, \ldots, x_{n}\right] \otimes\left[x_{n+1}, \ldots, x_{n+m}\right]\right)=\left[x_{1}, \ldots, x_{n+m}\right] .
$$

Скрешивающая коцепь $\alpha: K \rightarrow F K$ определена формулой $\alpha(x)=[x]$.

Дифференциал в алгебре $F K$ определяется условием скрешивающей коцепи $\alpha$ и имеет вид

$$
\begin{aligned}
d\left[x_{1}, \ldots, x_{n}\right]= & \sum_{i=1}^{n}(-1)^{\epsilon_{i-1}+1}\left[x_{1}, \ldots, d\left(x_{i}\right), \ldots, x_{n}\right] \\
& +\sum_{i=1}^{n}(-1)^{\epsilon_{i}}\left[x_{1}, \ldots, x_{i}^{\prime}, x_{i}^{\prime \prime}, \ldots, x_{n}\right]
\end{aligned}
$$

где $\nabla\left(x_{i}\right)=\sum x_{i}^{\prime} \otimes x_{i}^{\prime \prime}$.

Каждая скрешиваюшая коцепь $\phi: K \rightarrow A$ индуцирует отображение алгебр $F(\phi): F K \rightarrow A$,

$$
F(\phi)\left[x_{1}, \ldots, x_{n}\right]=\pi\left(\phi\left(x_{1}\right) \otimes \cdots \otimes \phi\left(x_{n}\right)\right) .
$$

С отображением коалгебр $B(\phi): K \rightarrow B A$ дело обстоит несколько сложнее. А именно, это отображение следует задавать формулой

$$
B(\phi)(x)=[\phi(x)]+\sum\left[\phi\left(x^{\prime}\right), \phi\left(x^{\prime \prime}\right)\right]+\sum\left[\phi\left(x^{\prime}\right), \phi\left(x^{\prime \prime}\right), \phi\left(x^{\prime \prime \prime}\right)\right]+\cdots,
$$

где $\sum x^{\prime} \otimes x^{\prime \prime}=\nabla(x), \sum x^{\prime} \otimes x^{\prime \prime} \otimes x^{\prime \prime \prime}=(\nabla \otimes 1) \nabla(x)$ и т.д. Однако в результате может получиться бесконечное число слагаемых. Избежать этого можно, наложив на коалгебру $K$ некоторые условия конечности. 
Будем говорить, что $K$ - коалгебра конечного типа, если для любого $x \in K$ найдется номер $n$, для которого $\nabla(n)(x)=0$, где $\nabla(n)=(\nabla \otimes \cdots \otimes 1) \ldots(\nabla \otimes 1) \nabla$ - итерация коумножения $\nabla$.

В этом случае мы будем иметь отображение коалгебр $B(\phi): K \rightarrow B A$. Если же коалгебра $K$ не такова, то рассмотрим фильтрацию $K^{1} \subset K^{2} \subset \cdots \subset K$, для которой

$$
K^{1}=\{x \in K \mid \nabla(x)=0\}, \quad \ldots, \quad K^{n}=\left\{x \in K \mid \nabla(x) \in \sum_{i=1}^{n-1} K^{i} \otimes K^{n-i}\right\}, \ldots
$$

Положим $\check{K}=\lim K^{n}$. Ясно, что $\check{K}-$ подкоалгебра коалгебры $K$ конечного типа и имеет место отображение коалгебр $B(\phi): \check{K} \rightarrow B A$.

В частности, скрещивающая коцеп $\alpha: K \rightarrow F K$ индуцирует отображение коалгебр $B(\alpha): \check{K} \rightarrow B F K$ и скрешиваюшая коцеп $\beta: B A \rightarrow A$ индуцирует отображение алгебр $F(\beta): F B A \rightarrow A$.

ПРЕДЛОЖЕНИЕ 1. Для произвольной алгебры А отображение

$$
F(\beta): F B A \rightarrow A
$$

является цепной эквивалентностью.

Обозначим для краткости отображение $F(\beta)$ через $\eta$ и определим отображение $\xi: A \rightarrow F B A$, положив $\xi(x)=[[x]]$. Ясно, что $\xi$ является цепным отображением (но не отображением алгебр) и $\eta \circ \xi=\mathrm{Id}$.

Покажем, что $\xi \circ \eta \simeq \mathrm{Id}: F B A \rightarrow F B A$. Для этого определим цепную гомотопию $h: F B A \rightarrow F B A$, положив

$$
h\left[\left[x_{1}\right],\left[x_{2}, \ldots, x_{n}\right], \ldots,\left[y_{1}, \ldots, y_{m}\right]\right]=\left[\left[x_{1}, \ldots, x_{n}\right], \ldots,\left[y_{1}, \ldots, y_{m}\right]\right]
$$

и $h(x)=0$ в остальных случаях.

Непосредственные вычисления показывают, что имеет место требуемая формула, т.е. $h$ является искомой цепной гомотопией.

ПРЕДЛОЖЕНИЕ $1^{\prime}$. Для произвольной коалгебры $K$ конечного типа отображение $B(\alpha): K \rightarrow B F K$ является цепной эквивалентностью.

Обозначим для краткости отображение $B(\alpha)$ через $\xi$ и определим отображение $\eta: B F K \rightarrow K$, положив $\eta[[x]]=x$ и $\eta(x)=0$ в остальных случаях. Ясно, что $\eta$ является цепным отображением (но не отображением коалгебр) и $\eta \circ \xi=\mathrm{Id}$. Покажем, что $\xi \circ \eta \simeq \mathrm{Id}$. Для этого определим цепную гомотопию $h: B F K \rightarrow$ $B F K$, положив

$$
h\left[\left[x_{1}, \ldots, x_{n}\right], \ldots,\left[y_{1}, \ldots, y_{m}\right]\right]=\left[\left[x_{1}\right],\left[x_{2}, \ldots, x_{n}\right], \ldots,\left[y_{1}, \ldots, y_{m}\right]\right], \quad n \geqslant 2,
$$

и 0 , если $n=1$.

Непосредственные вычисления показывают, что имеет место требуемая формула, т.е. $h$ является искомой цепной гомотопией. 
Рассмотрим теперь ситуацию, когда коалгебра $K$ не является коалгеброй конечного типа. Стандартная фильтрация в ко- $B$-конструкции $F K$ индуцирует фильтрацию в $B$-конструкции $B F K$. Обозначим через $\widehat{B} F K$ ее пополнение относительно этой фильтрации. Скрещивающая коцепь $\alpha: K \rightarrow F K$ индуцирует отображение коалгебр $\xi=\widehat{B}(\alpha): K \rightarrow \widehat{B} F K$. Кроме этого имеется проекция $\eta: \widehat{B} F K \rightarrow K$. Поскольку определенная выше гомотопия $h: B F K \rightarrow B F K$ сохраняет фильтрацию, она индуцирует гомотопию $h: \widehat{B} F K \rightarrow \widehat{B} F K, d(h)=\xi \circ \eta-$ Id. Следовательно, имеем следующее

ПРЕДЛОЖЕНИЕ $1^{\prime \prime}$. Для произвольной коалгебры $K$ отображение коалгебр $\widehat{B}(\alpha): K \rightarrow \widehat{B} F K$ является цепной әквивалентностью.

\section{§ 2. Модули и комодули}

Дифференциальный градуированный модуль $L$ называется левым комодулем над коалгеброй $K$, если задано отображение $\tau: L \rightarrow K \otimes L$, удовлетворяющее условию $(\nabla \otimes 1) \tau=(1 \otimes \tau) \tau$.

Дифференциальный градуированный модуль $M$ называется левым модулем над алгеброй $A$, если задано отображение $\mu: A \otimes M \rightarrow M$, удовлетворяюшее условию $\mu(\pi \otimes 1)=\mu(1 \otimes \mu)$.

Аналогично определяются правые модуль и комодуль.

Если $L$-правый $K$-комодуль, $M$ - левый $A$-модуль и $\phi: K \rightarrow A$-скрещивающая коцепь, то скрещенным тензорным произведением $L \otimes_{\phi} M$ называется дифференциальньй градуированный модуль, который как градуированный модуль совпадает с обычным тензорным произведением и в котором дифференциал $d_{\phi}$ определен формулой

$$
d_{\phi}=d \otimes 1+1 \otimes d+\phi \cap
$$

где $\phi \cap=(1 \otimes \mu)(1 \otimes \phi \otimes 1)(\tau \otimes 1): L \otimes M \rightarrow L \otimes M[2]$.

В частности, определены следуюшие скрещенные тензорные произведения: $K \otimes_{\phi} M, L \otimes_{\phi} A$ и $K \otimes_{\phi} A$.

Если $L$ - левый $K$-комодуль, $M$ - левьй $A$-модуль, $\phi: K \rightarrow A$ - скрещивающая коцепь, то скрещенным модулем гомоморфизмов $\operatorname{Hom}_{\phi}(L, M)$ называется дифференциальньй градуированньй модуль, который как градуированный модуль совпадает с обычным модулем гомоморфизмов и в котором дифференциал определен формулой $d_{\phi}(f)=d(f)+\phi \cup f, f \in \operatorname{Hom}(L, M)$.

Для левого $A$-модуля $M$ рассмотрим $B$-конструкцию $B(A, M)$, являющуюся левым комодулем над коалгеброй $B A$.

Образуюшие в $B(A, M)$ обозначаются через $\left[x_{1}, \ldots, x_{n}\right] x, x_{i} \in A, x \in M$, и имеют размерность, равную сумме размерностей элементов $x_{i}, x$ плюс $n$.

Дифференциал на этих элементах определен формулой

$$
\begin{aligned}
d\left(\left[x_{1}, \ldots, x_{n}\right] x\right)= & \sum_{i=1}^{n}(-1)^{\epsilon_{i-1}+1}\left[x_{1}, \ldots, d\left(x_{i}\right), \ldots, x_{n}\right] \\
& -(-1)^{\epsilon_{n}}\left[x_{1}, \ldots, x_{n}\right] d(x) \\
& +\sum_{i=1}^{n-1}(-1)^{\epsilon_{i}}\left[x_{1}, \ldots, \pi\left(x_{i} \otimes x_{i+1}\right), \ldots, x_{n}\right] \\
& +(-1)^{\epsilon_{n}}\left[x_{1}, \ldots, x_{n-1}\right] \mu\left(x_{n} \otimes x\right) .
\end{aligned}
$$


Аналогичным образом для правого $A$-модуля $M$ определяется $B$-конструкция $B(M, A)$, являющаяся правым $B A$-комодулем.

Двойственным образом для левого $K$-комодуля $L$ рассмотрим ко- $B$-конструкцию $F(K, L)$, являющуюся левым модулем над алгеброй $F K$.

Образующие в $F(K, L)$ обозначаются через $\left[x_{1}, \ldots, x_{n}\right] x, x_{i} \in K, x \in L$, и имеют размерность, равную сумме размерностей $x_{i}, x$ минус $n$.

Дифференциал на этих элементах определен формулой

$$
\begin{aligned}
d\left(\left[x_{1}, \ldots, x_{n}\right] x\right)= & \sum_{i=1}^{n}(-1)^{\epsilon_{i-1}+1}\left[x_{1}, \ldots, d\left(x_{i}\right), \ldots, x_{n}\right] x \\
& -(-1)^{\epsilon_{n}}\left[x_{1}, \ldots, x_{n}\right] d(x) \\
& +\sum_{i=1}^{n}(-1)^{\epsilon_{i}}\left[x_{1}, \ldots, x_{i}^{\prime}, x_{i}^{\prime \prime}, \ldots, x_{n}\right] x \\
& +(-1)^{\epsilon_{n+1}}\left[x_{1}, \ldots, x_{n}, x^{\prime}\right] x^{\prime \prime}
\end{aligned}
$$

где $\sum x_{i}^{\prime} \otimes x_{i}^{\prime \prime}=\nabla\left(x_{i}\right), \sum x^{\prime} \otimes x^{\prime \prime}=\tau(x)$.

Аналогично для правого $K$-комодуля $L$ определяется ко- $B$-конструкция $F(L, K)$, являюшаяся правым $F K$-модулем.

ПрЕДЛОЖЕНИЕ 2. Для произвольного левого модуля $M$ над алгеброй $A$ имеет место цепная эквивалентность $F(B A, B(A, M)) \simeq M$.

ПРЕДЛОЖЕНИЕ $2^{\prime}$. Для произвольного левого комодуля $L$ над коалгеброй конечного типа $K$ имеет место цепная әквивалентность

$$
B(F K, F(K, L)) \simeq L
$$

ПРЕДЛОЖЕНИЕ $2^{\prime \prime}$. Для произвольного левого комодуля $L$ над коалгеброй $K$ имеет место чепная эквивалентность $\widehat{B}(F K, F(K, L)) \simeq L$.

Доказательства проводятся аналогично доказательствам предложений $1,1^{\prime}$ и $1^{\prime \prime}$.

Аналогичные предложения имеют место для правых модулей и комодулей.

ПРЕДЛОЖЕНИЕ 3. Для произвольного правого А-модуля $M$ имеет место чепная эквивалентность правых $A$-модулей $B(M, A) \otimes_{\beta} A \simeq M$.

Определим отображение правых $A$-модулей $\eta: B(M, A) \otimes_{\beta} A \rightarrow M$, положив $\eta(x \otimes y)=\mu(x \otimes y), x \in M \subset B(M, A), y \in A$, и $\eta(x \otimes y)=0$ в остальных случаях. Ясно, что отображение $\eta$ является цепным, т.е. перестановочно с дифференциалом.

Определим цепное отображение $\xi: M \rightarrow B(A, M) \otimes_{\beta} A$, но не $A$-модульное отображение, положив $\xi(x)=x \otimes 1$. Ясно, что $\eta \circ \xi=$ Id. Покажем, что $\xi \circ \eta$ гомотопно тождественному отображению. Для этого определим цепную гомотопию $h: B(M, A) \otimes_{\beta} A \rightarrow B(M, A) \otimes_{\beta} A$, положив $h\left(x\left[x_{1}, \ldots, x_{n}\right] \otimes y\right)=$ $x\left[x_{1}, \ldots, x_{n}, y\right] \otimes 1$. Непосредственные вычисления показывают, что выполняется равенство $d(h)=\xi \circ \eta-\mathrm{Id}$ и, следовательно, $h$ является искомой цепной гомотопией.

Двойственным образом показывается, что имеет место следующее предложение. 
ПРЕДЛОЖЕНИЕ $3^{\prime}$. Для произвольного левого комодуля $L$ над коалгеброй $K$ имеет место иепная әквивалентность левых $K$-комодулей $K \otimes_{\alpha} F(K, L) \simeq L$.

Заметим, что скрешенное тензорное произведение $B(M, A) \otimes_{\beta} A$ изоморфно $B$-конструкции $B(M, A, A)$, являюшейся тотальным комплексом $A$-модульной резольвенты $M$. Двойственным образом скрещенное тензорное произведение $K \otimes_{\alpha} F(K, L)$ изоморфно ко- $B$-конструкции $F(K, K, L)$.

\section{§ 3. Модули и комодули Ли}

Здесь мы определим понятия модуля и комодуля Ли, которые в дальнейшем распространим на случай алгебр и коалгебр Ли над операдами.

ОПРЕДЕЛЕнИЕ 1. Дифференшиальный градуированньй модуль $X$ будем называть модулем Ли над коалгеброй $K$, если задано отображение $\phi: K \otimes X \rightarrow X$, понижающее размерность на единицу и удовлетворяющее условию скрешивающей коцепи $d(\phi)+\phi \cup \phi=0$, где $\phi \cup \phi=\phi(1 \otimes \phi)(\nabla \otimes 1): K \otimes X \rightarrow X$.

Пусть $M$ - левый модуль над алгеброй $A$ и $\psi: K \rightarrow A$ - скрешиваюшая коцеп. Тогда на $M$ можно задать структуру модуля Ли над коалгеброй $K$, $\phi: K \otimes M \rightarrow M$, положив $\phi=\mu(\psi \otimes 1)$. В частности, если $L-$ левый комодуль над коалгеброй $K$, то ко- $B$-конструкция $X=F(K, L)$ является левым модулем над алгеброй $F K$ и, следовательно, $X$ является $K$-модулем Ли. Таким образом, примеры модулей Ли дают рассмотренные ранее ко- $B$-конструкции.

Если $X$ - модуль Ли над коалгеброй $K$ и $L$ - правый $K$-комодуль, то в скрещенном тензорном произведении $L \otimes X$ можно определить новый дифференциал $d_{\phi}=d \otimes 1+1 \otimes d+\phi \cap$, преврашающий $L \otimes X$ в скрешенное тензорное произведение, обозначаемое $L \otimes_{\phi} X$. В частности, для коалгебры $K$ имеет место скрешенное тензорное произведение $K \otimes_{\phi} X$.

Если $X$ - модуль Ли над коалгеброй $K$ и $L$ - левый $K$-комодуль, то в модуле гомоморфизмов $\operatorname{Hom}(L, X)$ можно определить новый дифференциал $d_{\phi}, d_{\phi}(f)=$ $d(f)+\phi \cup f, f \in \operatorname{Hom}(L, X)$, превращающий $\operatorname{Hom}(L, X)$ в скрещенньй модуль гомоморфизмов, обозначаемьй $\operatorname{Hom}_{\phi}(L, X)$.

Таким образом, мы имеем функтор $F=F(K,-)$ из категории левых $K$-комодулей в категорию $K$-модулей Ли, который $K$-комодулю $L$ сопоставляет $K$-модуль Ли $F(K, L)$. Обозначим через $G$ функтор из категории $K$-модулей Ли в категорию $K$-комодулей, сопоставляющий $K$-модулю Ли $X$ комодуль $G(X)=K \otimes_{\phi} X$ над коалгеброй $K$. Имеет место естественное преобразование функторов $\alpha: \operatorname{Id} \rightarrow G \circ F$, $\alpha(L): L \rightarrow K \otimes_{\phi} F(K, L)$, которое, как следует из предложения $3^{\prime}$, является цепной эквивалентностью.

Аналогичньм образом показывается, что имеет место естественное преобразование функторов $\beta: F \circ G \rightarrow \mathrm{Id}, \beta(X): F\left(K, K \otimes_{\phi} X\right) \rightarrow X$, являюшееся цепной эквивалентностью.

Таким образом, функторы $F$ и $G$ являются сопряженными и имеет место

ПРЕДЛОЖЕНИЕ 4. Для коалгебры $K$ категория $K$-комодулей иепно әквивалентна категории $K$-модулей Ли.

Переформулировкой на язык модулей Ли теоремы Брауна [2] получается 
Теорема (Брауна). Для коалгебры $K$ конечного типа и произвольного $K$-модуля Ли $X, \phi: K \otimes X \rightarrow X$, на гомология $X_{*}=H_{*}(X)$ имеются структура $K$-модуля Ли $\phi_{*}: K \otimes X_{*} \rightarrow X_{*}$ и чепная әквивалентность $K$-комодулей $K \otimes_{\phi} X \simeq K \otimes_{\phi_{*}} X_{*}$.

Действительно, обозначим через $\xi: X_{*} \rightarrow X$ отображение выбора представителей в классах гомологий; $\eta: X \rightarrow X_{*}$ - обратное отображение, $\eta \circ \xi=\mathrm{Id}$; $h: X \rightarrow X$ - цепная гомотопия, $d(h)=\xi \circ \eta-\mathrm{Id}, h \circ \xi=0, \eta \circ h=0, h \circ h=0$.

Определим структуру $K$-модуля Ли на $X_{*}, \phi_{*}: K \otimes X_{*} \rightarrow X_{*}$, положив $\phi_{*}=$ $\eta \phi(1 \otimes \xi)+\eta \phi(1 \otimes h)(1 \otimes \phi)(\nabla \otimes 1)(1 \otimes \xi)+\cdots$.

Определим отображения $\xi_{*}: K \otimes_{\phi_{*}} X_{*} \rightarrow K \otimes_{\phi} X, \eta_{*}: K \otimes_{\phi} X \rightarrow K \otimes_{\phi_{*}} X_{*}$ и гомотопию $h_{*}: K \otimes_{\phi} X \rightarrow K \otimes_{\phi} X$, положив

$$
\begin{aligned}
\phi_{*} & =\eta \phi(1 \otimes \xi)+\eta \phi(1 \otimes h)(1 \otimes \phi)(\nabla \otimes 1)(1 \otimes \xi)+\cdots, \\
\xi_{*} & =1 \otimes \xi+(1 \otimes h)(1 \otimes \phi)(\nabla \otimes 1)(1 \otimes \xi)+\cdots, \\
\eta_{*} & =1 \otimes \eta+(1 \otimes \eta)(1 \otimes \phi)(1 \otimes 1 \otimes h)(\nabla \otimes 1)+\cdots, \\
h_{*} & =1 \otimes h+(1 \otimes h)(1 \otimes \phi)(1 \otimes 1 \otimes h)(\nabla \otimes 1)+\cdots .
\end{aligned}
$$

Непосредственные вычисления показывают, что справедливы требуемые формулы $\eta_{*} \circ \xi_{*}=\mathrm{Id}, d\left(h_{*}\right)=\xi_{*} \circ \eta_{*}-\mathrm{Id}, h_{*} \circ \xi=0, \eta \circ h_{*}=0, h_{*} \circ h_{*}=0$.

Заметим, что в этой теореме $X_{*}$ можно считать не только гомологиями $X$, а произвольным дифференциальным градуированньм модулем, цепно эквивалентным $X$, в смысле существования отображений $\xi: X_{*} \rightarrow X, \eta: X \rightarrow X_{*}$ и цепной гомотопии $h: X \rightarrow X$, удовлетворяющих перечисленным вьше условиям.

Рассмотрим теперь ситуацию, когда коалгебра $K$ не является коалгеброй конечного типа. В этом случае следует рассматривать $K$-модули Ли $X$ с фильтрацией $X=X^{1} \supset X^{2} \supset \cdots \supset X^{n} \supset \cdots$ такой, что $\phi\left(K \otimes X^{n}\right) \subset X^{n+1}$. Обозначим через $\widehat{X}$ пополнение $X$ относительно этой фильтрации.

Воспользуемся следуюшим вариантом теории возмушений дифференциальных градуированных модулей [3].

Теорема (Гугенхейма-Ламбе-Сташеффа). Пусть $X-$ дифференциальный градуированный модуль с фильтрачией $X=X^{1} \supset X^{2} \supset \cdots$, в котором дифференциал d представляется в виде суммы $d^{\prime}+d^{\prime \prime}$, где дифференциал $d^{\prime}$ сохраняет фильтрачию, а $d^{\prime \prime}$ увеличивает фильтрачию на единичу. Обозначим $\widehat{X}_{*}^{\prime}=H_{*}\left(\widehat{X}, d^{\prime}\right)$. Тогда на $\widehat{X}_{*}^{\prime}$ можно определить новый дифференциал $d_{*}^{\prime \prime}$, a также отображения $\xi: \widehat{X}_{*}^{\prime} \rightarrow \widehat{X}, \quad \eta: \widehat{X} \rightarrow \widehat{X}_{*}^{\prime}$ и гомотопию $h: \widehat{X} \rightarrow \widehat{X}$, удовлетворяющие перечисленным выше условиям.

Действительно, пусть $\xi^{\prime}: \widehat{X}_{*}^{\prime} \rightarrow \widehat{X}$ - отображение выбора представителей в классах гомологий, $\eta^{\prime}: \widehat{X} \rightarrow \widehat{X}_{*}^{\prime}, h^{\prime}: \widehat{X} \rightarrow \widehat{X}$ таковы, что $\eta^{\prime} \circ \xi^{\prime}=\mathrm{Id}, d^{\prime}\left(h^{\prime}\right)=$ $\xi^{\prime} \circ \eta^{\prime}-\mathrm{Id}, \eta^{\prime} \circ h^{\prime}=0, h^{\prime} \circ \xi^{\prime}=0, h^{\prime} \circ h^{\prime}=0$. Тогда искомый дифференциал $d_{*}^{\prime \prime}$, отображения $\xi: \widehat{X}_{*} \rightarrow \widehat{X}, \eta: \widehat{X} \rightarrow \widehat{X}_{*}$ и гомотопия $h: \widehat{X} \rightarrow \widehat{X}$ выражаются формулами

$$
\begin{aligned}
d_{*}^{\prime \prime} & =\eta^{\prime} d^{\prime \prime} \xi^{\prime}+\eta^{\prime} d^{\prime \prime} h^{\prime} d^{\prime \prime} \xi^{\prime}+\cdots, \\
\xi & =\xi^{\prime}+h^{\prime} d^{\prime \prime} \xi^{\prime}+h^{\prime} d^{\prime \prime} h^{\prime} d^{\prime \prime} \xi^{\prime}+\cdots, \\
\eta & =\eta^{\prime}+\eta^{\prime} d^{\prime \prime} h^{\prime}+\eta^{\prime} d^{\prime \prime} h^{\prime} d^{\prime \prime} h^{\prime}+\cdots, \\
h & =h^{\prime}+h^{\prime} d^{\prime \prime} h^{\prime}+h^{\prime} d^{\prime \prime} h^{\prime} d^{\prime \prime} h^{\prime}+\cdots .
\end{aligned}
$$

Из этого и теоремы Брауна получаем 
ПРЕДЛОЖЕНИЕ 5. Пусть $K-$ коалгебра, $X-K$-модуль Ли с фильтрацией $u$ дифференциалом $d=d^{\prime}+d^{\prime \prime}$, әде $d^{\prime}-$ дифференциал, сохраняющий фильтрачию, a $d^{\prime \prime}$ повышает фильтрацию на единичу. Тогда на $\widehat{X}_{*}^{\prime}=H_{*}\left(\widehat{X}, d^{\prime}\right)$ можно определить дифференциал $d_{*}^{\prime \prime}$ и структуру $K$-модуля Ли $\phi_{*}: K \otimes \widehat{X}_{*}^{\prime} \rightarrow \widehat{X}_{*}^{\prime}$, дающ,ую чепную әквивалентность $K$-комодулей $K \otimes_{\phi} \widehat{X} \simeq K \otimes_{\phi_{*}} \widehat{X}_{*}^{\prime}$.

Двойственным образом определим понятие комодуля Ли над алгеброй.

ОПРЕДЕЛЕНИЕ 1'. Дифференциальный градуированный модуль $Y$ будем называть комодулем Ли над алгеброй $A$, если задано отображение $\psi: Y \rightarrow Y \otimes A$, понижаюшее размерность на единицу и удовлетворяюшее условию скрешиваюшей коцепи $d(\psi)+\psi \cup \psi=0$.

Все перечисленные вьше свойства модулей Ли легко дуализируются на случай комодулей Ли. В частности, дуализация теоремы Брауна была рассмотрена нами в работе [4].

\section{§4. Коммутативные коалгебры и алгебры Ли}

Коалгебра $K$ с коумножением $\nabla: K \rightarrow K \otimes K$ назьвается коммутативной, если выполняется условие $T \nabla=\nabla$, где $T: K \otimes K \rightarrow K \otimes K$ - отображение перестановки сомножителей, $T(x \otimes y)=(-1)^{|x||y|} y \otimes x$.

Аналогично алгебра $A$ с умножением $\pi: A \otimes A \rightarrow A$ называется коммутативной, если $\pi T=\pi$.

В случае поля нулевой характеристики в работе [5] было показано, что категория коммутативных коалгебр гомотопически эквивалентна категории алгебр Ли. Здесь мы рассмотрим соотношение между коммутативными коалгебрами и алгебрами Ли, а также двойственную ситуацию в случае произвольного кольца коэффициентов.

Для модуля $X$ обозначим через $X \wedge X$ фактормодуль тензорного произведения $X \otimes X$ по отношению $x \otimes y+T(x \otimes y) \sim 0$. Аналогично обозначим через $X^{\wedge n}=$ $X \wedge \ldots \wedge X$ фактормодуль $X^{\otimes n}=X \otimes \cdots \otimes X$ по этим соотношениям.

ОПредЕлЕниЕ 2. Дифференциальный градуированный модуль $X$ будем называть $\wedge$-коалгеброй, если задано отображение $\nabla: X \rightarrow X \wedge X$, удовлетворяющее условию $(\nabla \wedge 1-1 \wedge \nabla) \nabla=0: X \rightarrow X \wedge X \wedge X$.

Двойственным образом для модуля $Y$ обозначим через $Y \vee Y$ подмодуль тензорного произведения $Y \otimes Y$, состоящий из элементов $z \in Y \otimes Y$, для которых $T(z)+z=0$, где $T: Y \otimes Y \rightarrow Y \otimes Y$ - отображение перестановки сомножителей. Аналогично определяется подмодуль $Y^{\vee n}$ тензорного произведения $Y^{\otimes n}$.

ОПРеДЕЛЕниЕ $2^{\prime}$. Дифференциальньй градуированньй модуль $Y$ будем называть $\vee$-алгеброй, если задано отображение $\pi: Y \vee Y \rightarrow Y$, удовлетворяющее условию $\pi(\pi \vee 1-1 \vee \pi)=0: Y \vee Y \vee Y \rightarrow Y$.

Если $K$ - обычная коалгебра, то коумножение $\nabla: K \rightarrow K \otimes K$ индуцирует коумножение $\nabla: K \rightarrow K \wedge K$, преврашая $K$ в $\wedge$-коалгебру.

Если $A$ - обычная алгебра, то умножение $\pi: A \otimes A \rightarrow A$ индуцирует умножение $\pi: Y \vee Y \rightarrow Y$, превращая $Y$ в $\vee$-алгебру.

Если $A$ - алгебра Хопфа с умножением $\pi$ и коумножением $\nabla$, то модуль $Q A=$ $A / \operatorname{Im} \pi$ ее неразложимых элементов будет являться $\wedge$-коалгеброй относительно 
коумножения $Q \nabla: Q A \rightarrow Q A \wedge Q A$, индуцированного коумножением $\nabla$. Аналогично модуль $P A=\operatorname{Ker} \nabla$ ее примитивных элементов будет являться $\vee$-алгеброй относительно умножения $P \pi: P A \vee P A \rightarrow P A$, индуцированного умножением $\pi$.

Если $R$ - поле характеристики, не равной 2 , то для модуля $Y$ имеет место отображение $\psi: Y \otimes Y \rightarrow Y \otimes Y, \psi(x \otimes y)=\frac{1}{2}\left(x \otimes y-(-1)^{|x||y|} y \otimes x\right)$. Если теперь $Y$ является $\vee$-алгеброй, то композиция $\pi \circ \psi$ задает умножение [ , ]: $Y \otimes Y \rightarrow Y$, удовлетворяюшее соотношениям

$$
\begin{gathered}
{[x, y]+(-1)^{|x||y|}[y, x]=0,} \\
{[[x, y], z]-(-1)^{|y||z|}[[x, z], y]+(-1)^{|x||y|+|x||z|}[[y, z], x]=0,}
\end{gathered}
$$

т.е. преврашает $Y$ в алгебру Ли. И наоборот, если $Y$ является алгеброй Ли с умножением [, ]: $Y \otimes Y \rightarrow Y$, то это умножение индуцирует отображение $\pi: Y \vee$ $Y \rightarrow Y$, преврашающее $Y$ в $\vee$-алгебру.

Таким образом, в случае поля характеристики, не равной 2 , понятия $\vee$-алгебры и алгебры Ли совпадают. Однако для наших целей даже в этом случае оказывается удобным пользоваться языком $\vee$-алгебр.

Иногда умножение в алгебре Ли удобно считать операцией, понижающей размерность на единицу. В этом случае определение $\vee$-алгебры также изменяется. A именно, в качестве $Y \vee Y$ следует рассматривать подмодуль тензорного произведения $Y \otimes Y$, состояший из всех устойчивых относительно перестановки сомножителей элементов. Аналогично при определении $\wedge$-коалгебры $X$, коумножение в которой понижает размерность на единицу, в качестве $X \wedge X$ следует рассматривать фактормодуль тензорного произведения $X \otimes X$ по действию симметрической групшы $\Sigma_{2}$.

Ясно, что если $Y-\vee$-алгебра, в которой умножение сохраняет размерность, то надстройка $\Sigma Y$ будет $\vee$-алгеброй, в которой умножение понижает размерность на единицу, и наоборот. Аналогично, если $X-\wedge$-коалгебра, в которой коумножение сохраняет размерность, то денадстройка $\Sigma^{-1} X$ будет $\wedge$-коалгеброй, в которой коумножение понижает размерность на единицу, и наоборот.

$\mathrm{B}$ дальнейшем мы, как правило, будем рассматривать $\vee$-алгебры и $\wedge$-коалгебры с сохраняющими размерности структурами.

Для $\vee$-алгебры $Y$ так же, как и для алгебры Ли, можно определить понятие универсальной обертывающей алгебры $U(Y)$ как факторалгебры тензорной алгебры $T Y$ по идеалу, порожденному элементами $i(z)-\pi(z)$, где $z \in Y \vee Y$, $i: Y \vee Y \rightarrow Y \otimes Y$ - вложение. Универсальность означает, что для любой алгебры $A$ и любого отображения $\vee$-алгебр $f: \underset{\sim}{Y} \rightarrow A$ сушествует единственное отображение алгебр $\widetilde{f}: U(Y) \rightarrow A$, для которого $\widetilde{f} \circ i=f$.

Если умножение в $\vee$-алгебре $Y$ тривиально, то универсальная обертываюшая алгебра $U(Y)$ является факторалгеброй тензорной алгебры по идеалу, порожденному элементами $i(z), z \in Y \vee Y$, т.е. такими элементами $z \in Y \otimes Y$, для которых $T(z)=-z$. Такую факторалгебру будем обозначать $W(Y)$.

Для универсальной обертывающей алгебры $U(Y)$ над $\vee$-алгеброй $Y$ стандартным образом определяются фильтрация

$$
U_{0}(Y)=R \subset U_{1}(Y) \subset \cdots \subset U_{n}(Y) \subset \cdots
$$


(см.[6]) и ассоциативная алгебра

$$
\operatorname{gr} U(Y)=\sum_{n \geqslant 0} \operatorname{gr}_{n} U(Y), \quad \operatorname{gr}_{n} U(Y)=U_{n}(Y) / U_{n-1}(Y)
$$

умножение в которой $\operatorname{gr}_{p} U(Y) \otimes \operatorname{gr}_{q} U(Y) \rightarrow \operatorname{gr}_{p+q} U(Y)$ индуцируется умножением в $U(Y)$.

Так же, как и для обычных алгебр Ли, показывается, что имеет место следующая теорема.

Теорема (Пуанкаре-Биркгофа-Витта). Каноническое отображение модулей $Y \rightarrow \operatorname{gr} U(Y)$ продолжается до отображения алгебр $W(Y) \rightarrow \operatorname{gr} U(Y)$, которое является изоморфизмом.

Двойственным образом для $\wedge$-коалгебры $X$ определяется универсальная обертывающая коалгебра $\cap(X)$ и рассматриваются соответствуюшие свойства.

Для модуля $M$ определим $\wedge$-коалгебру $\Lambda M=\sum \Lambda^{n} M$, положив $\Lambda^{1} M=M$, $\Lambda^{2} M=M \wedge M$ и $\nabla^{2}: \Lambda^{2} M \rightarrow \Lambda^{1} M \wedge \Lambda^{1} M$ - тождественное отображение. Предположим, что мы определили модули $\Lambda^{1} M, \ldots, \Lambda^{n-1} M$ и отображения $\nabla^{1}, \ldots, \nabla^{n-1}$. Рассмотрим отображение

$$
\nabla \wedge 1-1 \wedge \nabla: \quad \sum_{i=1}^{n-1} \Lambda^{i} M \wedge \Lambda^{n-i} M \rightarrow \sum_{i, j} \Lambda^{i} M \wedge \Lambda^{j} M \wedge \Lambda^{n-i-j} M
$$

и определим $\Lambda^{n} M$ как ядро этого отображения, а коумножение $\nabla^{n}$ как естественное вложение.

ПРЕДЛОЖЕНИЕ 6. ^-коалгебра $\Lambda$ является свободной $\wedge$-коалгеброй над $M$, т.е. для любой $\wedge$-коалгебры $X$ и любого отображения модулей $f: X \rightarrow M$ существует единственное отображение $\wedge$-коалгебр $\tilde{f}: X \rightarrow \Lambda M$, для которого $p \circ \tilde{f}=f$.

Положим $\tilde{f}^{1}=f: X \rightarrow M$. Предположим, что мы определили отображения $\tilde{f}^{1}, \ldots, \tilde{f}^{n-1}$. Для определения отображения $\tilde{f}^{n}: X \rightarrow \Lambda^{n} M$ рассмотрим отображение

$$
(\tilde{f} \wedge \tilde{f}) \nabla: \quad X \rightarrow \sum_{i=1}^{n-1} \Lambda^{i} M \wedge \Lambda^{n-i} M .
$$

Непосредственные вычисления показывают, что образ этого отображения лежит в ядре отображения $\nabla \wedge 1-1 \wedge \nabla$ и, следовательно, это отображение можно поднять до отображения $\tilde{f}^{n}: X \rightarrow \Lambda^{n} M$.

Двойственным образом для модуля $M$ можно определить $\vee$-алгебру $V(M)$, являющуюся свободной $\vee$-алгеброй над $M$.

В тензорной алгебре $T M$ рассмотрим коумножение $\nabla: T M \rightarrow T M \otimes T M$, преврашающее ее в кокоммутативную алгебру Хопфа, положив $\nabla(x)=x \otimes 1+1 \otimes x$ на образующих $x \in M$. Эта структура алгебры Хопфа в тензорной алгебре $T Y$ индуцирует структуру алгебры Хопфа в универсальной обертывающей алгебре $U(Y)$ над $\vee$-алгеброй $Y$. Двойственным образом для $\wedge$-коалгебры $X$ универсальная обертывающая коалгебра $\cap(X)$ имеет структуру коммутативной алгебры Хопфа. 
ПРЕДЛОЖЕНИЕ 7. Для модуля $M$ имеют место изоморфизмы алгебр Хопфa $U(V M) \cong T M, \cap(\Lambda M) \cong T M$.

Действительно, вложение $M \rightarrow T M$ индуцирует отображение $\vee$-алгебр $V M \rightarrow$ $T M$ и отображение алгебр $U(V M) \rightarrow T M$. С другой стороны, отображение $M \rightarrow U(V M)$ индуцирует отображение алгебр $T M \rightarrow U(V M)$, которое является взаимно обратным к отображению $U(V M) \rightarrow T M$.

Так же, как и в случае алгебр Ли, показывается, что для $\vee$-алгебры $Y$ модуль примитивных элементов $P U(Y)$ универсальной обертьвающей алгебры $U(Y)$, pacсматриваемой как алгебра Хопфа, изоморфен самой $\vee$-алгебре $Y$. Аналогично для универсальной обертьвающей коалгебры $\cap(X)$ над $\wedge$-коалгеброй $X$ модуль неразложимых элементов $Q \cap(X)$ изоморфен самой $\wedge$-коалгебре $X$.

Из предложения 7 отсюда следует

ПРЕДЛОЖЕНИЕ 8. Для модуля $M$ имеют место изоморфизмы $\vee$-алгебр и $\wedge$-коалгебр $V(M) \cong P T M, \Lambda M \cong Q T M$, где в первом случае TM рассматривается как кокоммутативная алгебра Хопфа, а во втором как коммутативная алгебра Хопфа.

\section{$\S 5 . \quad B$-конструкции для $\vee$-алгебр и $\wedge$-коалгебр}

Для коммутативной коалгебры $K$ обычная ко- $B$-конструкция $F K$ является алгеброй Хопфа, коумножение $\nabla: F K \rightarrow F K \otimes F K$ на образуюших $[x] \in F K$ задается формулой $\nabla([x])=1 \otimes[x]+[x] \otimes 1$. В силу сказанного выше модуль примитивных элементов $P F K$ этой алгебры Хопфа является $\vee$-алгеброй, которую мы будем обозначать $F_{\bigvee} K$ и называть $\vee$-ко-B-конструкцией над коммутативной коалгебpой $K$. Вложение $F_{\vee} K \rightarrow F K$ индуцирует отображение алгебр $U\left(F_{\vee} K\right) \rightarrow F K$, которое является изоморфизмом алгебр.

Двойственным образом для коммутативной алгебры $A$ обычная $B$-конструкция $B A$ является алгеброй Хопфа, умножение в которой задается формулой

$$
\left[x_{1}, \ldots, x_{n}\right] \cdot\left[x_{n+1}, \ldots, x_{n+m}\right]=\sum(-1)^{\epsilon}\left[x_{i_{1}}, \ldots, x_{i_{n+m}}\right],
$$

где сумма берется по всем $(n, m)$-перетасовкам чисел $1, \ldots, n+m, \epsilon$ - знак перетасовки.

Модуль неразложимых элементов $Q B A$ этой алгебры Хопфа является $\wedge$-коалгеброй, которую мы будем обозначать $B \wedge A$ и называть $\wedge$ - $B$-конструкиией над коммутативной алгеброй $A$. Проекция $B A \rightarrow B_{\wedge} A$ индуцирует отображение коалгебр $B A \rightarrow \cap\left(B_{\wedge} A\right)$, которое является изоморфизмом коалгебр.

Для $\vee$-алгебр и $\wedge$-коалгебр так же, как и для обычных алгебр и коалгебр, можно определить понятия скрешиваюшей коцепи и скрещенного тензорного произведения. А именно, пусть $X$-^-коалгебра и $A$ - коммутативная алгебра. Скрещивающей кочепью называется отображение $\phi: X \rightarrow A$, понижающее размерность на единицу и удовлетворяющее соотношению $d(\phi)+\phi \cup \phi=0$, где $\phi \cup \phi=\pi(\phi \wedge \phi) \nabla$. Для $\wedge-B$-конструкции $B \wedge A$ над коммутативной алгеброй $A$ имеет место стандартная скрешиваюшая коцепь $\beta: B \wedge A \rightarrow A$.

Если $M$ - модуль над коммутативной алгеброй $A, L$ - комодуль над $\wedge$-коалгеброй $X$ и $\phi: X \rightarrow A$ - скрещивающая коцеп, то скрещеннылм тензорныцм произведением называется дифференциальньй градуированный модуль $L \otimes_{\phi} M$, который 
как градуированный модуль совпадает с обычным тензорным произведением и в котором дифференциал $d_{\phi}$ определен формулой $d_{\phi}=d \otimes 1+1 \otimes d+\phi \cap$.

Двойственным образом для коммутативной коалгебры $K$ и $\vee$-алгебры $Y$ скрещивающей коцепью называется отображение $\phi: K \rightarrow Y$, понижающее размерность на единицу и удовлетворяющее условию $d(\phi)+\phi \cup \phi=0$, где $\phi \cup \phi=$ $\pi(\phi \vee \phi) \nabla$. Для $\vee$-ко- $B$-конструкции $F_{\vee} K$ над коммутативной коалгеброй $K$ имеет место стандартная скрещивающая коцепь $\alpha: K \rightarrow F_{\vee} K$.

Если $M$ - модуль над $\vee$-алгеброй $Y, L$ - комодуль над коммутативной коалгеброй $K$ и $\phi: K \rightarrow Y$ - скрещивающая коцеп, то скрещенным тензорны.м произведением называется дифференциальный градуированный модуль $L \otimes_{\phi} M$, которьй как градуированньй модуль совпадает с обычным тензорным произведением и в котором дифференщиал $d_{\phi}$ определен формулой $d_{\phi}=d \otimes 1+1 \otimes d+\phi \cap$.

Определим теперь понятия $B$-конструкции и ко- $B$-конструкции для $\vee$-алгебр и $\wedge$-коалгебр соответственно.

Пусть $X-\wedge$-коалгебра с коумножением $\nabla: X \rightarrow X \wedge X$. Рассмотрим свободную коммутативную алгебру $S \Sigma^{-1} X$ над денадстройкой над $X$ и определим в ней дифференциал, превращающий ее в коммутативную дифференциальную градуированную алгебру, положив

$$
d[x]=-[d(x)]-\sum(-1)^{\left|x^{\prime}\right|}\left[x^{\prime}, x^{\prime \prime}\right]
$$

где $\sum x^{\prime} \wedge x^{\prime \prime}=\nabla(x)$. На остальных элементах дифференциал определяется по формуле дифференциала от произведения. Полученную коммутативную дифференциальную градуированную алгебру будем называть ко-В-конструкцией над $X$ и обозначать $F X$.

Двойственным образом для $\vee$-алгебры $Y$ определяется $B$-конструкция $B Y$, являющаяся коммутативной дифференциальной градуированной коалгеброй, которая как градуированная коалгебра совпадает со свободной коммутативной коалгеброй $C \Sigma Y$ над надстройкой над $Y$.

Таким образом, мы имеем функтор $F_{\vee}$ из категории коммутативных коалгебр в категорию $\vee$-алгебр и функтор $B$ из категории $\vee$-алгебр в категорию коммутативных коалгебр.

Для коммутативной коалгебры $K$ конечного типа скрещивающая коцепь $\alpha: K \rightarrow$ $F_{\vee} K$ индуцирует отображение коалгебр $B(\alpha): K \rightarrow B F_{\vee} K$ и естественное преобразование функторов Id $\rightarrow B \circ F_{\vee}$.

Для $\vee$-алгебры $Y$ скрещивающая коцепь $\beta: B Y \rightarrow Y$ индуцирует отображение $\vee$-алгебр $F_{\vee}(\beta): F_{\vee} B Y \rightarrow Y$ и естественное преобразование функторов $F_{\vee} \circ B \rightarrow \mathrm{Id}$.

ПРЕДЛОЖЕНИЕ 9. Функторы $F_{\vee} u B$ являются сопряженными для категорий коммутативных коалгебр конечного типа $и \vee$-алгебр. Причем для коммутативной коалгебры $K$ конечного типа $u \vee$-алгебры $Y$ отображсения

$$
B(\alpha): K \rightarrow B F_{\vee} K, \quad F_{\vee}(\beta): F_{\vee} B Y \rightarrow Y
$$

являются иепными әквивалентностями.

Доказательство проводится аналогично доказательству предложения $1^{\prime}$. 
ПРЕДЛОЖЕНИЕ $9^{\prime}$. Функторы $B_{\wedge} u F$ являются сопряженными для ка-

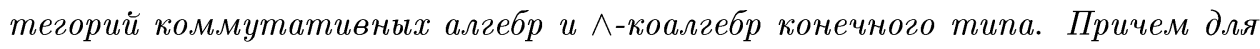
коммутативной алгебры $A$ и ^-коалгебры $X$ конечного типа отображения

$$
F(\beta): F B_{\wedge} A \rightarrow A, \quad B(\alpha): X \rightarrow B_{\wedge} F X
$$

являются иепными әквивалентностями.

В категории градуированных модулей рассмотрим функтор $S$, сопоставляющий модулю $M$ свободную коммутативную алгебру $S(M)$, порожденную $M$. Функтор $S$ является монадой и коммутативная алгебра $A$ является алгеброй над этой монадой. Поэтому имеет место $B$-конструкция

$$
B(S, A): A \leftarrow S(A) \leftarrow \cdots \leftarrow S^{n}(A) \leftarrow \cdots
$$

(см. [7]).

Двойственным образом функтор $C$, сопоставляющий модулю $M$ свободную коммутативную коалгебру $C(M)$, порожденную $M$, является комонадой, и коммутативная коалгебра $K$ является коалгеброй над этой комонадой. Поэтому имеет место ко- $B$-конструкция

$$
F(C, K): K \rightarrow C(K) \rightarrow \cdots \rightarrow C^{n}(K) \rightarrow \cdots .
$$

ПреДЛОЖенИЕ 10. Для коммутативных алгебры $А$ и коалгебры К конечного типа имеют место цепнье әквивалентности

$$
B(S, A) \simeq \Sigma^{-1} B_{\wedge} A, \quad F(C, K) \simeq \Sigma F_{\vee} K .
$$

Действительно, для коммутативной алгебры $A$ в силу предложения $9^{\prime}$ имеет место цепная эквивалентность $F B_{\wedge} A \simeq A$, которая индуцирует цепную эквивалентность $B\left(S, F B_{\wedge} A\right) \simeq B(S, A)$. Алгебра $F B_{\wedge} A$ как градуированная алгебра является свободной коммутативной алгеброй, порожденной $\Sigma^{-1} B_{\wedge} A$. Поэтому имеет место цепная эквивалентность $B(S, F B \wedge A) \simeq \Sigma^{-1} B \wedge A$. Следовательно, $B(S, A) \simeq B_{\wedge} A$. Аналогично показывается цепная эквивалентность $F(C, K) \simeq \Sigma F_{\vee} K$.

\section{§ 6. Операды и алгебры над операдами}

Напомним основные понятия, касающиеся операд и алгебр над операдами в категории дифференциальных градуированных модулей [7], [8].

Симметрическим семейством $\mathscr{E}=\{\mathscr{E}(j)\}_{j \geqslant 1}$ называется семейство дифференциальных градуированных модулей $\mathscr{E}(j)$, на которых действуют симметрические группы $\Sigma_{j}$. Отображсением симметрических семейств $f: \mathscr{E}^{\prime} \rightarrow \mathscr{E}^{\prime \prime}$ называется семейство $f=\{f(j)\}$ отображений $f(j): \mathscr{E}^{\prime}(j) \rightarrow \mathscr{E}^{\prime \prime}(j)$ дифференциальных градуированных модулей, перестановочных с действием симметрических групп.

Для симметрических семейств определены произведения $\mathscr{E}^{\prime} \otimes \mathscr{E}^{\prime \prime}, \mathscr{E}^{\prime} \times \mathscr{E}^{\prime \prime}$, для которых $\left(\mathscr{E}^{\prime} \otimes \mathscr{E}^{\prime \prime}\right)(j)=\mathscr{E}^{\prime}(j) \otimes \mathscr{E}^{\prime \prime}(j),\left(\mathscr{E}^{\prime} \times \mathscr{E}^{\prime \prime}\right)(j)-$ фактормодуль $\Sigma_{j}$-свободного модуля, порожденного модулем

$$
\sum_{j_{1}+\cdots+j_{k}=j} \mathscr{E}^{\prime}(k) \otimes \mathscr{E}^{\prime \prime}\left(j_{1}\right) \otimes \cdots \otimes \mathscr{E}^{\prime \prime}\left(j_{k}\right)
$$


по отношению эквивалентности , определяемому соотношениями

$$
\begin{aligned}
x^{\prime} \sigma \otimes x_{1}^{\prime \prime} \otimes \cdots \otimes x_{k}^{\prime \prime} & \sim x^{\prime} \otimes x_{\sigma^{-1}(1)}^{\prime \prime} \otimes \cdots \otimes x_{\sigma^{-1}(k)}^{\prime \prime} \sigma\left(j_{1}, \ldots, j_{k}\right), \\
x^{\prime} \otimes x_{1}^{\prime \prime} \sigma_{1} \otimes \cdots \otimes x_{k}^{\prime \prime} \sigma_{k} & \sim x^{\prime} \otimes x_{1}^{\prime \prime} \otimes \cdots \otimes x_{k}^{\prime \prime}\left(\sigma_{1} \times \cdots \times \sigma_{k}\right),
\end{aligned}
$$

где $\sigma \in \Sigma_{k}, \sigma_{i} \in \Sigma_{j_{i}}, 1 \leqslant i \leqslant k$ (см. [7], [8]).

Симметрическое семейство $\mathscr{E}$ называется операдой, если задано отображение симметрических семейств $\gamma: \mathscr{E} \times \mathscr{E} \rightarrow \mathscr{E}$, удовлетворяющее условию $\gamma(\gamma \times 1)=$ $\gamma(1 \times \gamma)$. Отображсением операд $f: \mathscr{E}^{\prime} \rightarrow \mathscr{E}^{\prime \prime}$ называется отображение симметрических семейств, удовлетворяющее условию $f \gamma^{\prime}=\gamma^{\prime \prime}(f \times f)$.

Основными примерами операд являются следуюшие.

1. Операда $E_{0}$, для которой $E_{0}(j)$ - свободный модуль с одной нульмерной образуюшей $e(j)$ и тривиальным действием симметрической группы $\Sigma_{j}$. Структура операды $\gamma: E_{0} \times E_{0} \rightarrow E_{0}$ задается формулой

$$
\gamma\left(e(k) \otimes e\left(j_{1}\right) \otimes \cdots \otimes e\left(j_{k}\right)\right)=e\left(j_{1}+\cdots+j_{k}\right)
$$

2. Операда $A$, для которой $A(j)$ - свободньй $\Sigma_{j}$-модуль, порожденный $E_{0}(j)$, и структура операды в $A$ индуцируется структурой операды в $E_{0}$.

3. Операда $A_{\infty}$, являющаяся $\Sigma_{j}$-свободной операдой, порожденной элементами $\pi_{n} \in A_{\infty}(n+2)$ размерности $n$, и дифференциал на этих элементах определен формулой

$$
d\left(\pi_{n+1}\right)=\sum_{i=0}^{n-1}(-1)^{(n+1)(k+1)+i k} \pi_{i}\left(1 \otimes \cdots \otimes \pi_{n-i} \otimes \cdots \otimes 1\right)
$$

где сумма берется также по всем местам $k$, на которых может стоять $\pi_{n-i}$.

4. Операда $E$, для которой $E(j)=\operatorname{Hom}\left(\Delta^{*} ; \Delta^{*} \otimes \cdots \otimes \Delta^{*}\right)$, где $\Delta^{*}=\left\{\Delta^{n}\right\}$-косимплициальный объект, состоящий из цепных комплексов стандартных $n$-мерных симплексов; Нот рассматривается в категории косимплищиальных объектов; действие симметрических груп определяется перестановками сомножителей в $\Delta^{*} \otimes \cdots \otimes \Delta^{*}$, и структура операды определена формулой

$$
\gamma\left(f \otimes g_{1} \otimes \cdots \otimes g_{k}\right)=\left(g_{1} \otimes \cdots \otimes g_{k}\right) f, \quad f \in E(k), \quad g_{i} \in E\left(j_{i}\right), \quad 1 \leqslant i \leqslant k .
$$

5. Операда $E_{\infty}$, являющаяся свободной, $\Sigma$-свободной и ацикличной операдой.

Двойственным образом симметрическое семейство $\mathscr{G}$ называется кооперадой, если задано отображение симметрических семейств $\delta: \mathscr{G} \rightarrow \mathscr{G} \times \mathscr{G}$, удовлетворяющее условию $(\delta \times 1) \delta=(1 \times \delta) \delta$. Отображсением кооперад $f: \mathscr{G}^{\prime} \rightarrow \mathscr{G}^{\prime \prime}$ называется отображение симметрических семейств, удовлетворяющее условию $\delta^{\prime \prime} f=(f \times f) \delta^{\prime}$.

Ясно, что если семейство $\mathscr{E}=\{\mathscr{E}(j)\}$, состоящее из конечно порожденных $\Sigma_{j}$-модулей $\mathscr{E}(j)$, является операдой, то двойственное семейство $\overline{\mathscr{E}}$, состоящее из двойственных $\Sigma_{j}$-модулей $\overline{\mathscr{E}}(j)=\operatorname{Hom}(\mathscr{E}(j), R)$, является кооперадой.

Симметрическое семейство $\mathscr{E}$ определяет функтор в категории дифференциальных градуированных модулей, который мы также будем обозначать через $\mathscr{E}$, по формуле

$$
\mathscr{E}(X)=\sum_{j \geqslant 1} \mathscr{E}(j) \otimes_{\Sigma_{j}} X^{\otimes j}
$$


Структура операды $\gamma: \mathscr{E} \times \mathscr{E} \rightarrow \mathscr{E}$ определяет структуру монады $\gamma: \mathscr{E} \circ \mathscr{E} \rightarrow \mathscr{E}$.

Дифференциальный градуированный модуль $X$ называется алгеброй над операдой $\mathscr{E}$, если он является алгеброй над соответствующей монадой, т.е. задано отображение $\mu: \mathscr{E}(X) \rightarrow X$, удовлетворяющее условиям

$$
\mu \circ(\gamma(X))=\mu \circ(\mathscr{E}(\mu)), \quad \mu \circ i=\operatorname{Id}: X \rightarrow X .
$$

Двойственным образом симметрическое семейство $\mathscr{G}$ определяет функтор в категории дифференциальных градуированных модулей, который мы будем обозначать через $\overline{\mathscr{G}}$, по формуле

$$
\overline{\mathscr{G}}(X)=\prod_{j \geqslant 1} \mathscr{G}(j) \otimes^{\Sigma_{j}} X^{\otimes j}
$$

Структура кооперады $\delta: \mathscr{G} \rightarrow \mathscr{G} \times \mathscr{G}$ определяет структуру комонады $\delta: \overline{\mathscr{G}} \rightarrow \overline{\mathscr{G}} \circ \overline{\mathscr{G}}$.

Дифференциальньй градуированный модуль $X$ назьвается коалzеброй над кооперадой $\mathscr{G}$, если он является коалгеброй над соответствующей комонадой $\overline{\mathscr{G}}$, т.е. задано отображение $\tau: X \rightarrow \overline{\mathscr{G}}(X)$, удовлетворяющее условиям

$$
\bar{\delta}(X) \circ \tau=\bar{G}(\tau) \circ \tau, \quad p \circ \tau=\mathrm{Id}: X \rightarrow X .
$$

Если операда $\mathscr{E}$ состоит из конечно порожденных $\Sigma_{j}$-модулей, то комонаду, соответствующую коопераде $\overline{\mathscr{E}}$, будем также обозначать $\overline{\mathscr{E}}$. Ясно, что

$$
\overline{\mathscr{E}}(X)=\prod_{j \geqslant 1} \operatorname{Hom}_{\Sigma_{j}}\left(\mathscr{E}(j), X^{\otimes j}\right) .
$$

Дифференциальный градуированньй модуль $X$ называется коалгеброй над операдой $\mathscr{E}$, если он является коалгеброй над соответствующей комонадой $\overline{\mathscr{E}}$.

Ясно, что алгебры (коалгебры) над операдой $E_{0}$ являются коммутативными алгебрами (коалгебрами). Алгебры (коалгебры) над операдой $A$ являются просто алгебрами (коалгебрами). Алгебры (коалгебры) над операдой $A_{\infty}$ называются $A_{\infty}$-алгебрами $\left(A_{\infty}-\right.$ коалгебрами) [9]. Алгебры (коалгебры) над операдой $E_{\infty}$ называются $E_{\infty}$-алгебрами $\left(E_{\infty}\right.$-коалгебрами) [7], [8].

Как было показано в работе [8], сингулярный цепной комплекс топологического пространства допускает естественную структуру коалгебры над операдой $E_{\infty}$.

\section{$\S 7 . \quad B$-конструкции для операд и алгебр над операдами}

Аналогия понятия операды с понятием алгебры позволяет определить для операды $\mathscr{E}$ понятие $B$-конструкции $B \mathscr{E}$. Образуюшие в $B \mathscr{E}$ будем обозначать $\left[x_{1}, \ldots, x_{n}\right]$, где $x_{1} \times \cdots \times x_{n} \in \mathscr{E}^{\times n}$, и их размерностью будем считать сумму размерностей $x_{i}, 1 \leqslant i \leqslant n$, плюс $n$. Дифференциал определяется так же, как и в обычной $B$-конструкции, только вместо обычного умножения участвует операдное умножение $\gamma$.

Двойственным образом для кооперады $\mathscr{G}$ определяется ко- $B$-конструкция $F \mathscr{G}$. Образуюшие в $F \mathscr{E}$ будем обозначать $\left[x_{1}, \ldots, x_{n}\right]$ и их размерностью будем считать сумму размерностей $x_{i}, 1 \leqslant i \leqslant n$, плюс $n$. Дифференциал определяется так же, 
как и в обычной ко- $B$-конструкции, только вместо обычного коумножения берется кооперадное коумножение $\delta$.

Выясним, какие структуры имеются на этих конструкциях. С этой целью для симметрического семейства $\mathscr{E}$ обозначим через $\mathscr{E} \vee \mathscr{E}$ подсемейство семейства $\mathscr{E} \times \mathscr{E}$, для которого $(\mathscr{E} \vee \mathscr{E})(j)$ порождается тензорными произведениями

$$
\mathscr{E}(k) \otimes \mathscr{E}\left(j_{1}\right) \otimes \cdots \otimes \mathscr{E}\left(j_{k}\right)
$$

в которых все $j_{i}, 1 \leqslant i \leqslant k$, за исключением одного, равны единище. Таким образом, семейство $\mathscr{E} \vee \mathscr{E}$ порождается элементами вида

$$
x \vee y=x \otimes 1 \otimes \cdots \otimes y \otimes \cdots \otimes 1
$$

Заметим, что так определенное произведение неассоциативно, т.е. нет естественного изоморфизма $\mathscr{E} \vee(\mathscr{E} \vee \mathscr{E}) \cong(\mathscr{E} \vee \mathscr{E}) \vee \mathscr{E}$. Имеется только вложение $\mathscr{E} \vee(\mathscr{E} \vee$ $\mathscr{E}) \subset(\mathscr{E} \vee \mathscr{E}) \vee \mathscr{E}$

ОПРЕДЕЛЕНИЕ 3. Семейство $\mathscr{E}$ будем называть $\vee$-операдой, если задано отображение $\gamma: \mathscr{E} \vee \mathscr{E} \rightarrow \mathscr{E}$, удовлетворяющее условию

$$
\gamma(1 \vee \gamma)=\gamma(\gamma \vee 1): \quad \mathscr{E} \vee(\mathscr{E} \vee \mathscr{E}) \rightarrow \mathscr{E}
$$

Ясно, что всякая операда является $\vee$-операдой, но не наоборот. Для установления обратной связи определим понятие коммутативной $\vee$-операды. Рассмотрим элементы вида

$$
x \vee y=(1 \otimes \cdots \otimes x(i) \otimes \cdots \otimes 1) \otimes(1 \otimes \cdots \otimes y(j) \otimes \cdots \otimes 1),
$$

где $x(i) \in \mathscr{E}(i)$ стоит на месте с номером $n_{i}, y(j) \in \mathscr{E}(j)$ стоит на месте с номером $m_{j}$. Перестановкой этого элемента будем считать тот же самый элемент, если выполняются неравенства $n_{i} \leqslant m_{j} \leqslant n_{i}+i-1$. Если же выполняется неравенство $m_{j}<n_{i}$ или неравенство $m_{j}>n_{i}+i-1$, то перестановкой этого элемента будем считать элемент $(-1)^{x y} y \vee x$,

$$
y \vee x=(1 \otimes \cdots \otimes y(j) \otimes \cdots \otimes 1) \otimes(1 \otimes \cdots \otimes x(i) \otimes \cdots \otimes 1),
$$

для которого в первом случае $y(j)$ стоит на месте с номером $m_{j}$ и $x(i)$ на месте с номером $n_{i}+j-1$. Во втором случае $y(j)$ стоит на месте с номером $m_{j}-i+1$ и $x(i)$ на месте с номером $n_{i}$.

ОПРЕДЕЛЕНИЕ 4. $\vee$-операду $\mathscr{E}$ будем называть коммутативной, если отображение $\gamma \circ(\gamma \vee 1):(\mathscr{E} \vee \mathscr{E}) \vee \mathscr{E} \rightarrow \mathscr{E}$ устойчиво относительно определенных вьше перестановок.

Ясно, что задание на семействе $\mathscr{E}$ структуры коммутативной $\vee$-операды равносильно заданию на $\mathscr{E}$ структуры операды.

Двойственным образом для симметрического семейства $\mathscr{G}$ обозначим через $\mathscr{G} \wedge$ $\mathscr{G}$ факторсемейство семейства $\mathscr{G} \times \mathscr{G}$, для которого $(\mathscr{G} \wedge \mathscr{G})(j)$ порождается тензорными произведениями $\mathscr{G}(k) \otimes \mathscr{G}\left(j_{1}\right) \otimes \cdots \otimes \mathscr{G}\left(j_{k}\right)$, для которых все $j_{i}, 1 \leqslant i \leqslant k$, за исключением одного, равны единище. Таким образом, семейство $\mathscr{G} \wedge \mathscr{G}$ порождается элементами вида $x \wedge y=x \otimes(1 \otimes \cdots \otimes y \otimes \cdots \otimes 1)$.

Заметим, что так определенное произведение неассоциативно, т.е. нет естественного изоморфизма $\mathscr{G} \wedge(\mathscr{G} \wedge \mathscr{G}) \cong(\mathscr{G} \wedge \mathscr{G}) \wedge \mathscr{G}$. Имеется только проекция $(\mathscr{G} \wedge \mathscr{G}) \wedge \mathscr{G} \rightarrow \mathscr{G} \wedge(\mathscr{G} \wedge \mathscr{G})$. 
ОПРЕДЕЛЕНИЕ $3^{\prime}$. Семейство $\mathscr{G}$ будем называть $\wedge$-кооперадой, если задано отображение $\delta: \mathscr{G} \rightarrow \mathscr{G} \wedge \mathscr{G}$, удовлетворяющее условию

$$
(1 \wedge \delta) \delta=(\delta \wedge 1) \delta: \mathscr{G} \rightarrow \mathscr{G} \wedge(\mathscr{G} \wedge \mathscr{G}) .
$$

ОПРЕДЕЛЕНИЕ $4^{\prime} . \wedge$-коопераду $\mathscr{G}$ будем называть коммутативной, если отображение $(\delta \wedge 1) \circ \delta: \mathscr{G} \rightarrow(\mathscr{G} \wedge \mathscr{G}) \wedge \mathscr{G}$ устойчиво относительно определенных выше перестановок.

Ясно, что задание на $\mathscr{G}$ структуры коммутативной $\wedge$-кооперады равносильно заданию на $\mathscr{G}$ структуры кооперады.

Легко видеть, что $B$-конструкция $B \mathscr{E}$ над операдой $\mathscr{E}$ является $\wedge$-кооперадой. Двойственным образом ко- $B$-конструкция $F \mathscr{G}$ над кооперадой $\mathscr{G}$ является $\vee$-операдой.

Рассмотрим теперь понятия $B_{\vee}$-конструкции и ко- $B_{\wedge}$-конструкции. Для этого для семейства $\mathscr{E}$ определим семейство $\mathscr{E} \vee \mathscr{E} \vee \mathscr{E}$ как подсемейство семейства $(\mathscr{E} \vee \mathscr{E}) \vee \mathscr{E}$, устойчивое относительно определенных выше перестановок элементов. Аналогичным образом определяется $\vee$-произведение большего числа сомножителей $\mathscr{E}^{\vee n}=\mathscr{E} \vee \cdots \vee \mathscr{E}$.

Двойственным образом для семейства $\mathscr{G}$ определяется семейство $\mathscr{G} \wedge \mathscr{G} \wedge \mathscr{G}$ как факторсемейство семейства $(\mathscr{G} \wedge \mathscr{G}) \wedge \mathscr{G}$ относительно определенных выше перестановок элементов. Аналогичным образом определяется $\wedge$-произведение большего числа сомножителей $\mathscr{G}^{\wedge}=\mathscr{G} \wedge \cdots \wedge \mathscr{G}$.

Для операды $\mathscr{E}$ определим $B_{\vee}$-конструкцию $B_{\vee} \mathscr{E}$ как подсемейство семейства $B \mathscr{E}$ относительно вложений $\mathscr{E}^{\vee n} \subset \mathscr{E}^{\times n}$. Легко видеть, что $B \vee \mathscr{E}$ является кооперадой.

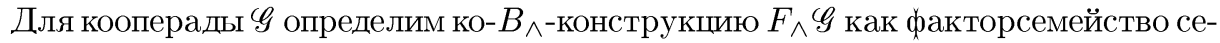
мейства $F \mathscr{G}$ относительно проекций $\mathscr{G} \times n \rightarrow \mathscr{G}^{\wedge}$. Легко видеть, что $F_{\wedge} \mathscr{G}$ является операдой.

ПРЕДЛОЖЕНИЕ 11. Для операды $\mathscr{E}$ вложение $B_{\vee} \mathscr{E} \subset B \mathscr{E}$ является цепной эквивалентностью. Двойственным образом для кооперады $\mathscr{E}$ проекиия $F \mathscr{E} \rightarrow$ $F_{\wedge} \mathscr{E}$ является иепной эквивалентностью.

Покажем, что вложение $B \vee \mathscr{E} \subset B \mathscr{E}$ является цепной эквивалентностью. Для этого определим фильтрацию в $B \mathscr{E}$, и тем самым в $B_{\vee} \mathscr{E}$, считая фильтрацию $1 \in$ $\mathscr{E}(1)$ равной нулю, фильтрацию элементов $x \in \mathscr{E}(j), j>1$, равной единище и фильтрацию элементов $x_{1} \times \cdots \times x_{n} \in \mathscr{E} \times \cdots \times \mathscr{E}$ равной сумме фильтраций элементов $x_{i}$. Исходное вложение $B_{\vee} \mathscr{E} \subset B \mathscr{E}$ сохраняет фильтрацию и индуцирует изоморфизм членов $E_{2}$ спектральных последовательностей. Следовательно, это вложение является цепной эквивалентностью.

Отметим, что хотя в общем случае $B$-конструкция $B \mathscr{E}$ над операдой $\mathscr{E}$ не обладает естественной структурой кооперады, тем не менее в некоторых случаях такая структура существует. Рассмотрим эти случаи. Обозначим через $\Delta^{*}=\left\{\Delta^{n}\right\}$ косимплициальный объект в категории дифференциальных градуированных модулей, состоящий из цепных комплексов $\Delta^{n}$ стандартных $n$-мерных симплексов. Предположим, что для операды $\mathscr{E}$ заданы перестановочные отображения $T: \Delta^{n} \otimes$ $\mathscr{E}(j) \rightarrow \mathscr{E}(j) \otimes \Delta^{n} \otimes \cdots \otimes \Delta^{n}$, согласованные с косимплициальной и операдной 
структурами. Эти перестановочные отображения будем кратко обозначать в виде $T: \Delta^{*} \times \mathscr{E} \rightarrow \mathscr{E} \times \Delta^{*}$.

$B$-конструкцию $B \mathscr{E}$ над операдой $\mathscr{E}$ можно представить в виде

$$
B \mathscr{E}=\sum_{n \geqslant 0} \Delta^{n} \otimes \mathscr{E}^{\times n} / \sim
$$

Искомая структура кооперады $\delta: B \mathscr{E} \rightarrow B \mathscr{E} \times B \mathscr{E}$ индуцируется отображениями

$$
\Delta^{n} \otimes \mathscr{E}^{\times n} \rightarrow \Delta^{i} \otimes \Delta^{n-i} \otimes \mathscr{E}^{\times i} \times \mathscr{E}^{\times n-i} \rightarrow\left(\Delta^{i} \otimes \mathscr{E}^{\times i}\right) \times\left(\Delta^{n-i} \otimes \mathscr{E}^{\times n-i}\right) .
$$

Двойственным образом если для кооперады $\mathscr{G}$ задано перестановочное отображение $T: \Delta^{*} \times \mathscr{G} \rightarrow \mathscr{G} \times \Delta^{*}$, то ко- $B$-конструкция $F \mathscr{G}$ является операдой.

ПРЕДЛОЖЕНИЕ 12. Функторы $B_{\vee} u F_{\wedge}$ являются сопряженными для категории операд и кооперад. Причем для операды $\mathscr{E}$ и кооперады $\mathscr{G}$ отображения

$$
F(\beta): F_{\wedge} B_{\vee} \mathscr{E} \rightarrow \mathscr{E}, \quad B(\alpha): G \rightarrow B_{\vee} F_{\wedge} G
$$

являются цепными эквивалентностями.

Доказательства требуемых цепных эквивалентностей проводятся аналогично доказательствам предложений 1 и $1^{\prime}$.

Пусть теперь $X$ - алгебра над операдой $\mathscr{E}$. Рассмотрим $B$-конструкции

$$
\begin{array}{r}
B(\mathscr{E}, X): X \leftarrow \mathscr{E} X \leftarrow \mathscr{E}^{\times 2} X \leftarrow \cdots \leftarrow \mathscr{E}^{\times n} X \leftarrow \cdots, \\
B_{\vee}(\mathscr{E}, X): X \leftarrow \mathscr{E} X \leftarrow \mathscr{E}^{\vee 2} X \leftarrow \cdots \leftarrow \mathscr{E}^{\vee n} X \leftarrow \cdots .
\end{array}
$$

Двойственным образом для коалгебры $Y$ над кооперадой $\mathscr{G}$ рассмотрим ко- $B$-конструкции

$$
\begin{gathered}
F(\mathscr{G}, Y): Y \rightarrow \mathscr{G} Y \rightarrow \mathscr{G}^{\times 2} Y \rightarrow \cdots \rightarrow \mathscr{G}^{\times n} Y \rightarrow \cdots \\
F_{\wedge}(\mathscr{G}, Y): Y \rightarrow \mathscr{G} Y \rightarrow \mathscr{G}^{\wedge 2} Y \rightarrow \cdots \rightarrow \mathscr{G}^{\wedge n} Y \rightarrow \cdots .
\end{gathered}
$$

Аналогично предложению 11 доказьвается

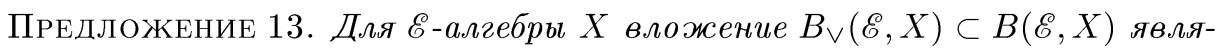
ется иепной эквивалентностью. Двойственным образом для $\mathscr{G - к о а л г е б р ь ~}$ проекция $F(\mathscr{G}, Y) \rightarrow F_{\wedge}(\mathscr{G}, Y)$ является цепной әквивалентностью.

\section{§ 8. Алгебры Ли над операдами}

По аналогии с понятиями $A_{\infty}$-алгебры и $A_{\infty}$-коалгебры [9] определим понятия $A_{\infty}$-У-алгебры и $A_{\infty}$-^-коалгебры.

ОПРЕДЕЛЕНИЕ 5. Дифференциальный градуированный модуль $Y$ будем называть $A_{\infty}-\vee$-алгеброй, если заданы отображения $\pi_{i}: Y^{\vee(i+2)} \rightarrow Y, i \geqslant 0$, повьшающие размерность на $i$ и удовлетворяюшие соотношениям

$$
d\left(\pi_{n+1}\right)=\sum_{i=0}^{n}(-1)^{(n+1)(k+1)+i k} \pi_{i}\left(1 \otimes \cdots \otimes \pi_{n-i} \otimes \cdots \otimes 1\right)
$$


где сумма берется также по всем местам $k$, на которых может стоять $\pi_{n-i}$.

Например, при $n=0$ имеет место соотношение

$$
d\left(\pi_{1}\right)=\pi_{0}\left(\pi_{0} \otimes 1-1 \otimes \pi_{0}\right),
$$

означающее гомотопическую ассоциативность отображения $\pi_{0}$.

Как и в случае $A_{\infty}$-алгебр, задание такого семейства отображений $\pi_{i}$ равносильно заданию дифференциала в $B$-конструкции $B Y$, который превращает $B Y$ в коммутативную дифференциальную коалгебру.

Заметим, что обычные дифференциальные $\vee$-алгебры можно рассматривать как $A_{\infty}-\vee$-алгебры, в которых высшие умножения $\pi_{i}, i \geqslant 1$, равны нулю.

Пусть $Y^{\prime}, Y^{\prime \prime}-A_{\infty}$-V-алгебры. $A_{\infty}$-отображсением из $Y^{\prime}$ в $Y^{\prime \prime}$ будем называть семейство отображений $\xi_{i}: Y^{\prime \vee}(i+1) \rightarrow Y^{\prime \prime}, i \geqslant 0$, повьшаюших размерность на $i$ и удовлетворяющих соотношениям

$$
\begin{aligned}
d\left(\xi_{n+1}\right)= & \sum_{i=0}^{n}(-1)^{(n+1)(k+1)+k i} \xi_{i}\left(1 \otimes \cdots \otimes \pi_{n-i}^{\prime} \otimes \cdots \otimes 1\right) \\
& +\sum_{i=0}^{n}(-1)^{n_{2}+n_{4}+\cdots} \pi_{i}^{\prime \prime}\left(\xi_{n_{1}} \otimes \cdots \otimes \xi_{n_{i+2}}\right),
\end{aligned}
$$

где $k$ - номер места, на котором стоит $\pi_{n-i}^{\prime}, n_{1}+\cdots+n_{i+2}=n-i$.

Например, если $n=0$, то имеем соотношение

$$
d\left(\xi_{1}\right)=\xi_{0} \pi_{0}^{\prime}-\pi_{0}^{\prime \prime}\left(\xi_{0} \otimes \xi_{0}\right)
$$

означающее гомотопическую мультипликативность отображения $\xi_{0}$.

Задание $A_{\infty}$-отображения $\xi$ из $Y^{\prime}$ в $Y^{\prime \prime}$ равносильно заданию отображения дифференщиальных коалгебр $B \xi: B Y^{\prime} \rightarrow B Y^{\prime \prime}$.

ОПРЕДЕЛЕНИЕ $5^{\prime}$. Дифференциальный градуированный модуль $X$ будем называть $A_{\infty}$-^-коалгеброй, если заданы отображения $\nabla_{i}: X \rightarrow X^{\wedge(i+2)}, i \geqslant 0$, повышаюшие размерность на $i$ и удовлетворяюшие соотношениям

$$
d\left(\nabla_{n+1}\right)=\sum_{i=0}^{n}(-1)^{(n+1)(k+1)+i(k+n-i)}\left(1 \otimes \cdots \otimes \nabla_{n-i} \otimes \cdots \otimes 1\right) \nabla_{i}
$$

Как и в случае $A_{\infty}$-коалгебр, задание такого семейства отображений $\nabla_{i}$ равносильно заданию дифференциала в ко- $B$-конструкции $F X$, которьй превращает $F X$ в коммутативную дифференциальную алгебру.

ПРЕДЛОЖЕНИЕ 14. Если основное кольио $R$ является полем характерис-

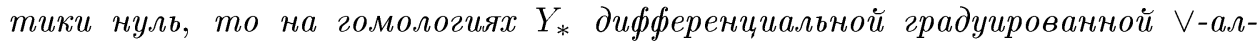
гебры $Y$ имеется структура $A_{\infty}-\bigvee$-алгебрь и цепная эквивалентность коалгебр $B Y \simeq B Y_{*}$. Двойственным образом на гомологиях $X_{*}$ дифференциальной градуированной $\wedge$-коалгебры $X$ имеется структура $A_{\infty}$-^-коалгебры и иепная әквивалентность алгебр $\widehat{F} X \simeq \widehat{F} X_{*}$.

Доказательство проводится так же, как и для обычных дифференциальных алгебр и коалгебр [10]. Условие на основное кольцо $R$ объясняется необходимостью, чтобы имели место изоморфизмы $H_{*}\left(Y^{\vee n}\right) \cong Y_{*}^{\vee n}, H_{*}\left(X^{\wedge n}\right) \cong X_{*}^{\wedge n}$.

Напомним также определения коммутативных $A_{\infty}$-алгебр и $A_{\infty}$-коалгебр (см. [11]). 
ОПРЕДЕЛЕНИЕ $6 . A_{\infty}$-коалгебра $K$ называется коммутативной $A_{\infty}$-коалгеброй, если отображения $\nabla_{i}: K \rightarrow K^{\otimes(i+2)}, i \geqslant 0$, повышаюшие размерность на $i$, составляющие структуру $A_{\infty}$-коалгебры, дополнительно удовлетворяют соотношениям коммутативности, т.е. для любых $n \geqslant 0$ и $1 \leqslant i \leqslant n+1$ выполняется равенство

$$
\sum_{i=1}^{n+1}(-1)^{\epsilon} \sigma(i, n+2-i) \nabla_{n}=0,
$$

где сумма берется по всем $(i, n+2-i)$-перетасовкам $\sigma, \epsilon-$ знак перетасовки.

Соотношения коммутативности означают, что операции $\nabla_{n}$ определяют на $F_{\vee} K$ структуру диффференциальной градуированной $\vee$-алгебры.

ОПредЕЛЕНИЕ $6^{\prime} . A_{\infty}$-алгебра $A$ называется коммутативной $A_{\infty}$-алгеброй, если отображения $\pi_{i}: A^{\otimes(i+2)} \rightarrow A, i \geqslant 0$, повышающие размерность на $i$, составляющие структуру $A_{\infty}$-алгебры, дополнительно удовлетворяют соотношениям коммутативности, т.е. для любых $n \geqslant 0$ и $1 \leqslant i \leqslant n+1$ выполняется равенство

$$
\sum_{i=1}^{n+1}(-1)^{\epsilon} \pi_{n} \sigma(i, n+2-i)=0
$$

где сумма берется по всем $(i, n+2-i)$-перетасовкам $\sigma, \epsilon-$ знак перетасовки.

Соотношения коммутативности означают, что операции $\pi_{n}$ определяют на $B \wedge A$ структуру дифференциальной градуированной $\wedge$-коалгебры.

ПРЕДЛОЖЕНИЕ $14^{\prime}$. Если основное кольцо $R$ является полем характеристики нуль, то на гомологиях $A_{*}$ коммутативной дифференциальной градуированной алгебры А имеется структура коммутативной $A_{\infty}$-алгебры и иепная әквивалентность $\wedge$-коалгебр $B \wedge A \simeq B_{\wedge} A_{*}$. Двойственным образом на гомологиях $K_{*}$ коммутативной дифференциальной градуированной коалгебры К имеется структура коммутативной $A_{\infty}$-коалгебры и чепная әквивалентность $\vee$-алгебр $\widehat{F}_{\vee} K \simeq \widehat{F}_{\vee} K_{*}$.

Определения коммутативных $A_{\infty}$-алгебр и $A_{\infty}$-коалгебр можно переформулировать и на языке операд. А именно, обозначим через $S_{\infty}=\left\{S_{\infty}(j)\right\}$ операду, являющуюся фактором операды $A_{\infty}$, по соотношениям

$$
\sum_{i=1}^{n+1}(-1)^{\epsilon} \pi_{n} \sigma(i, n+2-i)=0
$$

Тогда коммутативная $A_{\infty}$-алгебра является алгеброй над операдой $S_{\infty}$, а коммутативная $A_{\infty}$-коалгебра является коалгеброй над операдой $S_{\infty}$.

В случае, если основное кольцо $R$ является полем рациональных чисел $Q$ на сингулярном комплексе цепей $C_{*}(X, Q)$, имеется структура коммутативной $A_{\infty}$-коалгебры [11]. Функтор, сопоставляющий односвязному топологическому пространству $X$ дифференциальную градуированную $\vee$-алгебру $F_{\vee} C_{*}(X, Q)$, аналогичен функтору Квиллена [5], и гомологии $H_{*}\left(F_{\vee} C_{*}(X, Q)\right)$ изоморфны рациональным гомотопическим группам $\pi_{*-1}(X) \otimes Q$. 
ОпРЕдЕЛЕниЕ 7. Дифференциальный градуированный модуль $Y$ будем называть алгеброй Ли над кооперадой $\mathscr{G}$, если задано отображение $\phi: \overline{\mathscr{G}}(Y) \rightarrow Y$, понижающее размерность на единицу и удовлетворяющее условию скрещивающей коцепи $d(\phi)+\phi \cup \phi=0$, где $\phi \cup \phi=\phi(\overline{\mathscr{G}}(\phi) \delta(Y))$. В случае если $\mathscr{G}$ является кооперадой, двойственной к операде $\mathscr{E}$, т.е. $\mathscr{G}=\overline{\mathscr{E}}$, алгебры Ли над кооперадой $\overline{\mathscr{E}}$ будем называть также алгебрами Ли над операдой $\mathscr{E}$.

Из определения $A_{\infty}$ - $\mathrm{V}$-алгебры непосредственно следует, что задание на $Y$ структуры $A_{\infty}-\bigvee$-алгебры равносильно заданию на $\Sigma Y$ структуры алгебры Ли над операдой $E_{0}$.

Для нас наиболее важную роль будут играть алгебры Ли над операдой $E_{\infty}$ или просто $E_{\infty}$-алгебры Ли. Проекция $p: E_{\infty} \rightarrow E_{0}$ индуцирует отображение $\bar{E}_{0}(Y) \rightarrow \bar{E}_{\infty}(Y)$, и если $Y$ является $E_{\infty}$-алгеброй Ли, то $Y$ будет также являться $E_{0}$-алгеброй Ли.

Примеры алгебр Ли над операдой или кооперадой дают ко- $B$-конструкции. А именно, если $X$ является коалгеброй над кооперадой $\mathscr{G}$, то ко- $B$-конструкция $F_{\wedge}(\mathscr{G}$, $X)$ является $\mathscr{G}$-алгеброй Ли. В частности, если $\mathscr{G}$ является кооперадой, двойственной к операде $\mathscr{E}$, и $X$ - коалгебра над операдой $\mathscr{E}$, то $F_{\wedge}(\overline{\mathscr{E}}, X)$ будет алгеброй Ли над операдой $\mathscr{E}$.

Ко- $B$-конструкция $F(\mathscr{G}, X)$, вообще говоря, не является $\mathscr{G}$-алгеброй Ли. Однако если задано перестановочное отображение $T: \Delta^{*} \times \mathscr{G} \rightarrow \mathscr{G} \times \Delta^{*}$, то ко- $B$-конструкция $F(\mathscr{G}, X)$ уже будет алгеброй Ли над кооперадой $\mathscr{G}$. В дальнейшем мы будем рассматривать именно такие кооперады.

Таким образом, ко- $B$-конструкция дает нам функтор $F=F(\mathscr{G},-)$ из категории

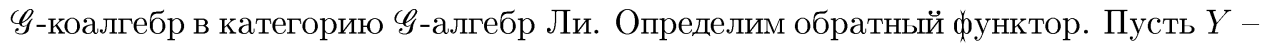
алгебра Ли над кооперадой $\mathscr{G}$. Тогда в $\overline{\mathscr{G}}(Y)$ можно определить новый дифференциал $d_{\phi}=d \times 1+1 \times d+\phi \cap$, превращающий $\overline{\mathscr{G}}(Y)$ в $\mathscr{G}$-коалгебру.

Вложение $i: X \rightarrow F(\mathscr{G}, X)$ индуцирует отображение коалгебр над кооперадой $\mathscr{G}, \alpha(X): X \rightarrow \overline{\mathscr{G}}_{\phi}(F(\mathscr{G}, X))$. Проекция $p: \overline{\mathscr{G}}_{\phi}(Y) \rightarrow Y$ индуцирует отображение

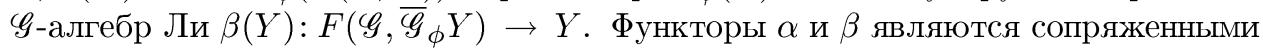
для категории $\mathscr{G}$-коалгебр и $\mathscr{G}$-алгебр Ли. Аналогично тому, как это было для категорий $K$-комодулей и $K$-модулей Ли, отображения $\alpha(X)$ и $\beta(Y)$ являются цепньми эквивалентностями, и, следовательно, имеет место

ПРЕДЛОЖЕНИЕ 15. Категория коалгебр над кооперадой $\mathscr{G}$ цепно әквивалентна категории $\mathscr{G - а л г е б р ~ Л и . ~ В ~ ч а с т н о с т и , ~ к а т е г о р и я ~ к о а л г е б р ~ н а д ~ о п е - ~}$ радой $\mathscr{E}$ чепно әквивалентна категории $\mathscr{E}$-алгебр Ли.

\section{Аналогично предложению 5 имеет место}

ПРЕДЛОЖЕНИЕ 16. Пусть $\mathscr{G}$ - кооперада, $Y-\mathscr{G}$-алгебра Ли с фильтрачией и дифференциалом $d=d^{\prime}+d^{\prime \prime}$, где $d^{\prime}-$ дифференциал, сохраняющий фильтрацию, а d" повыциет фильтрацию на единичу. Тогдана $\widehat{Y}_{*}^{\prime}=H_{*}\left(\widehat{Y}, d^{\prime}\right)$ мож-

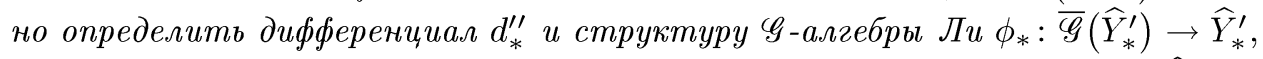

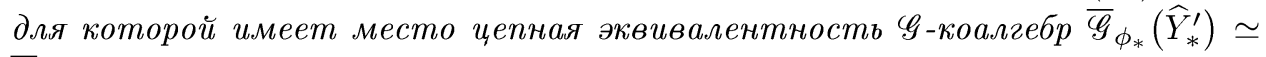
$\overline{\mathscr{G}}_{\phi}(Y)$.

Двойственным образом определим понятие коалгебры Ли над операдой. 
ОПРЕДЕЛЕНИЕ $7^{\prime}$. Дифференциальный градуированный модуль $Y$ будем называть коалгеброй Ли над операдой $\mathscr{E}$, если задано отображение $\psi: Y \rightarrow \mathscr{E}(Y)$, понижаюшее размерность на единицу и удовлетворяющее условию $d(\psi)+\psi \cup \psi=0$.

Рассмотренные выше свойства алгебр Ли легко дуализируются на случай коалгебр Ли.

\section{§9. Гомотопическая теория $E_{\infty}$-алгебр Ли}

Как было показано в предыдущем параграфе, категория $E_{\infty}$-алгебр Ли цепно эквивалентна категории $E_{\infty}$-коалгебр. В работе [12] для $E_{\infty}$-коалгебр была построена гомотопическая теория, позволяюшая свести гомотопическую теорию топологических пространств к гомотопической теории $E_{\infty}$-коалгебр. Здесь мы покажем, что гомотопическая теория $E_{\infty}$-коалгебр эквивалентна гомотопической теории $E_{\infty}$-алгебр Ли. Но перед этим рассмотрим более простую ситуацию комодулей и модулей Ли.

Пусть $Z, X$ - комодули над коалгеброй $K$. Слабым отображением $K$-комодулей $\tilde{f}: Z \rightsquigarrow X$ называется отображение $K$-комодулей $f: Z \rightarrow F(K, K, X)$. Два слабых отображения $\tilde{f}, \tilde{g}: Z \rightsquigarrow X$ называются гомотопными (обозначается $\tilde{f} \simeq \tilde{g}$ ), если сушествует слабое отображение $\tilde{h}: Z \rightsquigarrow X$, повышающее размерность на единищу и такое, что $d(\tilde{h})=\tilde{g}-\tilde{f}$. Модуль гомотопических классов слабых отображений $K$-комодулей $\tilde{f}: Z \rightsquigarrow X$ по введенному отношению гомотопности обозначим $[Z, X]$.

Пусть теперь $Y-K$-модуль Ли. Скрещенным отображсением $f: Z \rightarrow Y$ будем называть отображение, сохраняющее размерность, для которого выполняется равенство $d(f)+\phi \cup f=0$. Скрешенные отображения из $Z$ в $Y$ образуют модуль, который мы будем обозначать $\operatorname{Hom}_{\phi}(Z, Y)$.

Для скрешенных отображений $f, g: Z \rightarrow Y$ определим отношение гомотопности $\simeq$, положив $f \simeq g$, если сушествует отображение $h: Z \rightarrow Y$, повьшаюшее размерность на единицу и такое, что $d(h)+\phi \cup h=g-f$. Модуль гомотопических классов скрешенных отображений $f: Z \rightarrow Y$ будем обозначать $[Z, Y]_{\phi}$.

Из теоремы Брауна следует

ПРЕДЛОЖЕНИЕ 17. Если $K$ - коалгебра конечного типа, $Z-K$-комодуль, $Y-K$-модуль Ли, то на гомологиях $Y_{*}=H_{*}(Y)$ имеются структура $K$-модуля Ли и изоморфизм $[Z, Y]_{\phi} \cong\left[Z, Y_{*}\right]_{\phi_{*}}$.

Если коалгебра $K$ не является коалгеброй конечного типа, то рассмотрим $K$-модуль Ли $Y$ с фильтрацией и дифференциалом $d=d^{\prime}+d^{\prime \prime}$, для которого $d^{\prime}$ сохраняет фильтрацию, а $d^{\prime \prime}$ повьшает фильтрацию на единицу. Из предложения 5 следует, что в этом случае имеет место изоморфизм $[Z, \widehat{Y}]_{\phi} \cong\left[Z, \widehat{Y}_{*}\right]_{\phi_{*}}$.

Легко видеть, что задание скрешенного отображения $f: Z \rightarrow Y$ равносильно заданию $K$-комодульного отображения $\tilde{f}: Z \rightarrow K \otimes_{\phi} Y$ и задание гомотопии $h: Z \rightarrow Y$ между $f$ и $g$ равносильно заданию гомотопии $\tilde{h}: Z \rightarrow K \otimes_{\phi} Y$ между отображениями $\tilde{f}$ и $\tilde{g}$. Учитывая изоморфизм $K \otimes_{\alpha} F(K, X) \cong F(K, K, X)$, получаем, что задание слабого отображения $K$-комодулей $\tilde{f}: Z \rightsquigarrow X$ равносильно заданию скрешенного отображения $f: Z \rightarrow F(K, X)$ и задание гомотопии между слабыми отображениями $K$-комодулей равносильно заданию гомотопии между соответствуюшими скрешенными отображениями. Таким образом, имеет место следующее 
ПРЕДЛОЖЕНИЕ 18. Для комодулей $Z$ и $X$ над коалгеброй $K$ имеет место изоморфизм гомотопических классов $[Z, X] \cong[Z, F(K, X)]_{\phi}$.

Пусть теперь $X-E_{\infty}$-коалгебра, $\tau: X \rightarrow \bar{E}_{\infty} X, \quad \gamma: E_{\infty} \times E_{\infty} \rightarrow E_{\infty}$. Рассмотрим ко- $B$-конструкцию $F\left(E_{\infty}, E_{\infty}, X\right)$, являюшуюся тотальным комплексом $E_{\infty}$-коалгебрной резольвенты $X$. Слабым отображсением $E_{\infty}$-коалгебр $\tilde{f}$ : $Z \rightsquigarrow X$ будем называть отображение $E_{\infty}$-коалгебр $\tilde{f}: Z \rightarrow F\left(E_{\infty}, E_{\infty}, X\right)$. Два слабых отображения $\tilde{f}, \tilde{g}: Z \rightsquigarrow X$ называются гомотопными (обозначается $\tilde{f} \simeq \tilde{g})$, если существует слабое отображение $\tilde{h}: \Delta^{1} \otimes Z \rightsquigarrow X$ такое, что $\tilde{h}\left(\delta^{0} \otimes 1\right)=\tilde{f}$ и $\tilde{h}\left(\delta^{1} \otimes 1\right)=\tilde{g}$. Множество гомотопических классов слабых $E_{\infty}$-коалгебрных отображений из $Z$ в $X$ обозначим $[Z, X]$.

В работе [12] было показано что для односвязных топологических пространств $X_{1}$ и $X_{2}$ множество гомотопических классов $\left[X_{1}, X_{2}\right]$ непрерывных отображений топологических пространств находится в биективном соответствии с множеством гомотопических классов $\left[C_{*}\left(X_{1}\right), C_{*}\left(X_{2}\right)\right]$ слабых $E_{\infty}$-коалгебрных отображений их сингулярных цепных комплексов.

Пусть $Y-E_{\infty}$-алгебра Ли, $Z-E_{\infty}$-коалгебра. Скрещенным отображениeм $f: Z \rightarrow Y$ будем называть отображение, сохраняюшее размерность и такое, что $d(f)+\phi \cup f=0$. Заметим, что в отличие от ситуации, рассмотренной вьше, множество скрещенных отображений не образует модуль. Для скрещенных отображений $f, g: Z \rightarrow Y$ определим отношение гомотопности $\simeq$, положив $f \simeq g$, если сушествует отображение $h: Z \rightarrow Y$ размерности единица такое, что $d(h)+\phi \cup h=g-f$. Множество гомотопических классов скрешенных отображений $f: Z \rightarrow Y$ будем обозначать $[Z, Y]_{\phi}$.

Из предложения 16 следует

ПРеДЛОЖЕНИЕ 19. Пусть $Z-E_{\infty}$-коалгебра и $Y-E_{\infty}$-алгебра Ли с фильтрацией и дифференциалом $d=d^{\prime}+d^{\prime \prime}$, для которого $d^{\prime}$ сохраняет фильтраиию, а $d^{\prime \prime}$ повыишает фильтрацию на единичу. Тогда имеет место биекиия $[Z, \widehat{Y}]_{\phi} \longleftrightarrow\left[Z, \widehat{Y}_{*}\right]_{\phi_{*}}$, где $\widehat{Y}_{*}=H_{*}(\widehat{Y}), \widehat{Y}-$ пополнения $Y$.

Легко видеть, что задание скрешенного отображения $f: Z \rightarrow Y$ равносильно заданию $E_{\infty}$-коалгебрного отображения $\tilde{f}: Z \rightarrow \bar{E}_{\infty \phi}(Y)$, и задание гомотопии $h: Z \rightarrow Y$ между $f$ и $g$ равносильно заданию гомотопии $\tilde{h}: Z \rightarrow \bar{E}_{\infty \phi}(Y)$ между отображениями $\tilde{f}$ и $\tilde{g}$. Учитывая изоморфизм $\bar{E}_{\infty \alpha} F\left(E_{\infty}, X\right) \cong F\left(E_{\infty}, E_{\infty}, X\right)$, получаем, что задание слабого отображения $E_{\infty}$-коалгебр $\tilde{f}: Z \rightsquigarrow X$ равносильно заданию скрешенного отображения $f: Z \rightarrow F\left(E_{\infty}, X\right)$ и задание гомотопии между слабыми отображениями $E_{\infty}$-коалгебр равносильно заданию гомотопии между соответствуюшими скрещенныги отображениями. Таким образом, имеет место следующее

ПРЕДЛОЖЕНИЕ 20. Для коалгебр $Z$ и $X$ над операдой $E_{\infty}$ имеет место биекиия гомотопических классов $[Z, X] \longleftrightarrow\left[Z, F\left(E_{\infty}, X\right)\right]_{\phi}$.

Рассмотрим стандартную фильтрацию в ко- $B$-конструкции $Y=F\left(E_{\infty}, X\right)$ и выгислим гомологии $Y_{*}=H_{*}\left(Y, d^{\prime}\right)$.

Как было показано в работе [13], в случае $R=\mathbb{Z} / 2$ имеет место изоморфизм $H_{*}\left(\bar{E}_{\infty}(X)\right) \cong C \overline{\mathscr{E}}_{*}\left(X_{*}\right)$, где $X_{*}=H_{*}(X) ; \overline{\mathscr{E}}_{*}\left(X_{*}\right)$ - градуированный модуль, порожденньй элементами вида ${\overline{e_{1} \ldots e_{i}}}_{i_{k}} x_{n}, 0 \leqslant i_{1} \leqslant \cdots \leqslant i_{k}$, размерности $n 2^{k}-i_{1}-2 i_{2}-\cdots-2^{k-1} i_{k} ; C \overline{\mathscr{E}}_{*}\left(X_{*}\right)-$ свободная коммутативная коалгебра, 
порожденная $\overline{\mathscr{E}}_{*} X_{*}$, профакторизованная по соотношениям $\bar{e}_{0} x=x \otimes x$. Таким образом, $C \overline{\mathscr{E}}_{*}\left(X_{*}\right)$ является свободной коммутативной коалгеброй, порожденной элементами ${\overline{e_{i_{1}} \ldots e_{i}}}_{i_{k}} x_{n}$, для которых $1 \leqslant i_{1} \leqslant \cdots \leqslant i_{k}$.

Обозначим для краткости $C \overline{\mathscr{E}}_{*}\left(X_{*}\right)$ через $\bar{E}_{*}\left(X_{*}\right)$. Ясно, что как градуированный модуль $Y_{*}$ изоморфен ко- $B$-конструкции $F\left(\bar{E}_{*}, X_{*}\right)$. Как следует из предложений 16 и 20 , в этой ко- $B$-конструкции можно определить дифференциал $d_{*}^{\prime \prime}$ и структуру $E_{\infty}$-алгебры Ли $\phi_{*}$ так, что для $E_{\infty}$-коалгебры $Z$ будут иметь место биекции $[Z, X] \longleftrightarrow\left[Z, F\left(E_{\infty}, X\right)\right]_{\phi} \longleftrightarrow\left[Z, F\left(\bar{E}_{*}, X_{*}\right)\right]_{\phi_{*}}$. В частности, если $Z=S^{n-}$ цепной комплекс $n$-мерной сферы, то имеет место биекция

$$
\left[S^{n}, X\right]=\pi_{n}(X) \longleftrightarrow\left[S^{n}, F\left(\bar{E}_{*}, X_{*}\right)\right]_{\phi_{*}}
$$

Определим в $F\left(\bar{E}_{*}, X_{*}\right)$ новый дифференциал $\widetilde{d}_{*}$, положив

$$
\widetilde{d}_{*}(y)=d_{*}^{\prime \prime}(y)+\phi_{*} \cup \bar{y}
$$

где $d_{*}^{\prime \prime}$ - старый дифференциал в $F\left(\bar{E}_{*}, X_{*}\right), y \in F\left(\bar{E}_{*}, X_{*}\right), \operatorname{dim}(y)=n, \bar{y}: S^{n} \rightarrow$ $F\left(\bar{E}_{*}, X_{*}\right)$ - характеристическое отображение. Ко- $B$-конструкцию $F\left(\bar{E}_{*}, X_{*}\right)$ с таким дифференциалом $\widetilde{d}_{*}$ обозначим $\widetilde{F}\left(\bar{E}_{*}, X_{*}\right)$. Из определения множества $\left[S^{n}\right.$, $\left.F\left(\bar{E}_{*}, X_{*}\right)\right]$ непосредственно следует, что имеет место биекция $\left[S^{n}, F\left(\bar{E}_{*}, X_{*}\right)\right] \longleftrightarrow$ $H_{n}\left(\widetilde{F}\left(\bar{E}_{*}, X_{*}\right)\right)$, и, следовательно, имеем

ПРЕДЛОЖЕНИЕ 21. Для односвязного топологического пространства $X$ гомотопические әруппь $\pi_{*}(X)$, точнее, член $E^{\infty}$ их спектральной последовательности, изоморфны гомологиям ко-В-конструкиии $\widetilde{F}\left(\bar{E}_{*}, X_{*}\right)$, где $X_{*}=$ $H_{*}(X)$.

Отметим, что дифференциал в ко- $B$-конструкции $\widetilde{F}\left(\bar{E}_{*}, X_{*}\right)$ выражается через высшие гомологические операции [14], а само предложение 21 является переформулировкой соответствующего утверждения работы [15].

Выясним теперь вопрос о структуре $E_{\infty}$-алгебры Ли на $\widetilde{F}\left(\bar{E}_{*}, X_{*}\right)$. Скрещивающая коцепь $\phi_{*}: \bar{E}_{\infty} F\left(\bar{E}_{*}, X_{*}\right) \rightarrow F\left(\bar{E}_{*}, X_{*}\right)$ будет индуцировать отображение $\phi_{*}: \bar{E}_{\infty} \widetilde{F}\left(\bar{E}_{*}, X_{*}\right) \rightarrow \widetilde{F}\left(\bar{E}_{*}, X_{*}\right)$. Оно, конечно, не будет скрешивающей коцепю в старом понимании. Подправим определение скрещивающей коцепи с тем, чтобы устранить этот недостаток.

Для градуированного модуля $Y_{*}$ определим отображение $i: Y_{*} \rightarrow \bar{E}_{\infty}\left(Y_{*}\right)$, положив $i\left(y_{n}\right)=\bar{E}_{\infty}\left(\bar{y}_{n}\right) \tau\left(u_{n}\right)$, где $\bar{y}_{n}: S^{n} \rightarrow Y_{*}-$ характеристическое отображение для $y_{n} \in Y_{n}, u_{n}$ - образуюшая в $S^{n}, \tau: S^{n} \rightarrow \bar{E}_{\infty}\left(S^{n}\right)$ - структура $E_{\infty}$-коалгебры на $S^{n}$. Для $\phi_{*}: \bar{E}_{\infty}\left(Y_{*}\right) \rightarrow Y_{*}$ определим теперь новое $\cup$-произведение, положив $\phi_{*} \cup \phi_{*}=\phi_{*}\left(\bar{E}_{\infty} \phi_{*}\right)\left(\bar{\gamma} Y_{*}\right)+\phi_{*}\left(\bar{E}_{\infty} \phi_{*}\right) \bar{E}_{\infty}(i)$, где первое слагаемое в этой сумме соответствует старому $\cup$-произведению. Относительно нового $\cup$-произведения отображение $\phi_{*}: \bar{E}_{\infty}\left(\widetilde{F}\left(\bar{E}_{*}, X_{*}\right)\right) \rightarrow \widetilde{F}\left(\bar{E}_{*}, X_{*}\right)$ уже будет скрешивающей коцепью, и, следовательно, оно задает на $\widetilde{F}\left(\bar{E}_{*}, X_{*}\right)$ структуру $E_{\infty}$-алгебры Ли, для которой имеет место цепная эквивалентность $E_{\infty}$-коалгебр $\bar{E}_{\infty \phi_{*}} \widetilde{F}\left(\bar{E}_{*}, X_{*}\right) \simeq X_{*}$. Переходя к гомологиям, получаем следующую теорему. 
Теорема 1. Гомологии ко-В-конструкиии $\widetilde{F}\left(\bar{E}_{*}, X_{*}\right)$, а следовательно, и гомотопические группы $\pi_{*}(X)$ односвязного топологического пространства $X$ (точнее, член $E^{\infty}$ спектральной последовательности Боусфилда-Кана [16]) являются $E_{\infty}$-алгеброй Ли. Причем имеет место иепная әквивалентность $E_{\infty}$-коалгебр $\bar{E}_{\infty \phi_{*}}\left(\pi_{*}(X)\right) \simeq X_{*}=H_{*}(X)$.

Поскольку структура $E_{\infty}$-коалгебры на гомологиях топологического пространства определяет его слабый гомотопический тип, то получаем, что структура $E_{\infty}$-алгебры Ли на гомотопических группах топологического пространства (точнее, на члене $E^{\infty}$ спектральной последовательности) определяет слабый гомотопический тип этого пространства.

\section{$\S 10$. Описание $A_{\infty}$-алгебр и коалгебр}

Здесь мы распространим метод описания обычных $A_{\infty}$-алгебр и коалгебр [15] на случай $A_{\infty}-\vee$-алгебр и $A_{\infty}$-^-коалгебр. Основное кольцо будем предполагать полем характеристики нуль.

Пусть $Y$ - градуированная $A_{\infty}$ - $\vee$-алгебра. Последовательность $\left(y^{2}, \ldots, y^{n}\right)$ элементов $y^{i} \in Y^{\vee i}$ будем называть последовательностью Масси [17], если выполнены соотношения

$$
\begin{aligned}
& \left(\pi_{0} \otimes \cdots \otimes 1-\cdots+(-1)^{n} 1 \otimes \cdots \pi_{0}\right)\left(y^{n}\right)=0, \\
& \left(\pi_{1} \otimes \cdots \otimes 1+\cdots+1 \otimes \cdots \otimes \pi_{1}\right)\left(y^{n}\right) \\
& \quad \quad=\left(\pi_{0} \otimes \cdots \otimes 1-\cdots+(-1)^{n} 1 \otimes \cdots \otimes \pi_{0}\right)\left(y^{n-1}\right), \\
& \quad \cdots \cdots \cdots \cdots \cdots \cdots \cdots \cdots \cdots \cdots \cdots \cdots \cdots \cdots \cdots \cdots \cdots \cdots \cdots \cdots \cdots+\left(\pi_{0} \otimes 1-1 \otimes \pi_{0}\right)\left(y^{3}\right) . \\
& \quad\left(\pi_{n-3} \otimes 1+1 \otimes \pi_{n-3}\right)\left(y^{n}\right) \\
& \quad=\left(\pi_{n-4} \otimes 1+(-1)^{n} 1 \otimes \pi_{n-4}\right)\left(y^{n-1}\right)+\cdots+
\end{aligned}
$$

Для таких последовательностей Масси $\left(y^{2}, \ldots, y^{n}\right)$ определим произведение Масси $\mu\left(y^{2}, \ldots, y^{n}\right)=\pi_{0}\left(y^{2}\right)+\cdots+\pi_{n-2}\left(y^{n}\right)$. Элемент $y \in Y$ назовем разложимым, если он является образом какого-нибудь произведения Масси. Фактор $Y$ по разложимым элементам называется модулем неразложимых әлементов и обозначается $J Y$.

Переведем это определение на язык $B$-конструкций. Рассмотрим $B$-конструкцию $B Y$ над $A_{\infty}$-V-алгеброй $Y$. Вложение $i: Y \rightarrow B Y$ индуцирует короткую точную последовательность

$$
0 \rightarrow Y \stackrel{i}{\rightarrow} B Y \stackrel{p}{\rightarrow} B^{1} Y=B Y / Y \rightarrow 0
$$

и длинную точную последовательность гомологий

$$
\cdots \rightarrow Y \stackrel{i_{*}}{\longrightarrow} H_{*}(B Y) \stackrel{p_{*}}{\longrightarrow} H_{*}\left(B^{1} Y\right) \stackrel{\mu_{*}}{\longrightarrow} Y \rightarrow \cdots .
$$

Из определения последовательности Масси следует, что последовательность $\left(y^{2}, \ldots, y^{n}\right)$ является последовательностью Масси в том и только том случае, когда элемент $y=y^{2}+\cdots+y^{n}$ является циклом в $B^{1}(Y)$. Более того, отображение $\mu_{*}$ индуцируется произведением Масси. Таким образом, элемент $y \in Y$ 
разложим тогда и только тогда, когда он принадлежит образу $\mu_{*}$. Следовательно, модуль неразложимых элементов $J Y$ изоморфен образу отображения $i_{*}: Y \rightarrow H_{*}(B Y)$.

Для нахождения соотношений нужно указать такие последовательности Масси $\left(y^{2}, \ldots, y^{n}\right)$, произведения Масси $\mu\left(y^{2}, \ldots, y^{n}\right)$ от которых равны нулю. Часть таких соотношений следует непосредственно из структуры $A_{\infty}-\vee$-алгебры. Так же, как и в случае обычных $A_{\infty}$-алгебр, показывается, что это происходит в случаях, если циклы $y^{2}+\cdots+y^{n} \in B^{1} Y$ гомологичны нулю. Таким образом, соотношения, которые не следуют просто из структуры $A_{\infty}-\vee$-алгебры в $Y$, определяются ядром гомоморфизма $\mu_{*}: H_{*}\left(B^{1} Y\right) \rightarrow Y$ или, что то же самое, образом гомоморфизма $p_{*}: H_{*}(B Y) \rightarrow H_{*}\left(B^{1} Y\right)$.

Двойственным образом для градуированной $A_{\infty}$-^-коалгебры $X$ рассмотрим ко- $B$-конструкцию $F X$. Проекция $p: F X \rightarrow X$ индуцирует короткую точную последовательность

$$
0 \rightarrow F^{1} X \stackrel{i}{\rightarrow} F X \stackrel{p}{\rightarrow} X \rightarrow 0
$$

и длинную точную последовательность гомологий

$$
\cdots \rightarrow H_{*}\left(F^{1} X\right) \stackrel{i_{*}}{\longrightarrow} H_{*}(F X) \stackrel{p_{*}}{\longrightarrow} X \stackrel{\nu_{*}}{\longrightarrow} H_{*}\left(F^{1} X\right) \rightarrow \cdots .
$$

Элемент $x \in X$ называется примитивным, если он принадлежит ядру гомоморфизма $\nu_{*}: X \rightarrow H_{*}\left(F^{1} X\right)$ или, что то же самое, образу гомоморфизма $p_{*}$ : $H_{*}(F X) \rightarrow X$. Модуль примитивных элементов $A_{\infty}$-^-коалгебры $X$ обозначим $P X$.

Аналогичным образом, используя $B_{\wedge}$-конструкцию и ко- $B-\vee$-конструкцию, можно определить понятие разложимых элементов и найти соотношения для коммутативной градуированной $A_{\infty}$-алгебры, а также понятие примитивных элементов для коммутативной градуированной $A_{\infty}$-коалгебры.

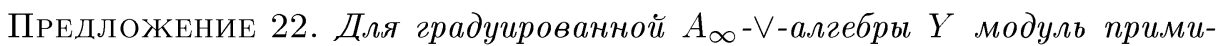
тивных әлементов $P H_{*}(B Y)$ гомологий $B$-конструкиии ВY изоморфен модулю неразложимых әлементов $A_{\infty}-\vee$-алгебры $Y: P H_{*}(B Y) \cong J Y$.

Действительно, по определению модуля примитивных элементов имеем

$$
P H_{*}(B Y)=\operatorname{Im}\left\{H_{*}\left(F_{\vee} H_{*}(B Y)\right) \rightarrow H_{*}(B Y)\right\} .
$$

Используя изоморфиим $H_{*}\left(F_{\vee} H_{*}(B Y)\right) \cong Y$, получаем изоморфизм $P H_{*}(B Y) \cong$ $\operatorname{Im}\left\{Y \rightarrow H_{*}(B Y)\right\}$. Легко видеть, что этот образ совпадает с образом гомоморфизма $Y \rightarrow H_{*}(B Y)$, который по определению дает модуль неразложимых элементов $A_{\infty}-\vee$-алгебры $Y$.

Двойственным образом имеем

ПРЕДЛОЖЕНИЕ $22^{\prime}$. Для произвольной градуированной $A_{\infty}$-^-коалгебрь $X$ модуль неразложимых әлементов $J H_{*}(F X)$ изоморфен модулю примитивных әлементов $P X A_{\infty}$-^-коалгебры $X$.

Применяя предложение 22 к рациональным гомотопическим группам, получаем

ПРЕДЛОЖЕНИЕ 23. Для рациональных гомотопических групп $\pi_{*}(X) \otimes Q$ односвязного топологического пространства $X$, рассматриваемых как $A_{\infty}-\vee$ алгебра, модуль неразложимиых әлементов $J\left(\pi_{*}(X) \otimes Q\right)$ изоморфен образу гомоморфизма Гуревича $H: \pi_{*}(X) \otimes Q \rightarrow H_{*}(X, Q)$. Соотношения определяются образом гомоморфизма $p_{*}: H_{*}(X, Q) \rightarrow H_{*}\left(B^{1}\left(\pi_{*}(X) \otimes Q\right)\right)$. 


\section{$\S 11$. Описание модулей и алгебр Ли}

Наша задача состоит в определении понятия разложимых элементов и соотношений для $E_{\infty}$-алгебр Ли, а также в их нахождении для гомотопических групп топологических пространств. Но прежде рассмотрим более простую ситуацию модулей Ли над коалгеброй.

Пусть $K$ - коалгебра, $\nabla: K \rightarrow K \otimes K, Y$ - градуированный модуль Ли над коалгеброй $K, \phi: K \otimes Y \rightarrow Y$. Вложение $i: Y \rightarrow K \otimes_{\phi} Y$ индуцирует короткую точную последовательность

$$
0 \rightarrow Y \stackrel{i}{\rightarrow} K \otimes_{\phi} Y \stackrel{p}{\rightarrow} K \otimes_{\phi}^{1} Y \rightarrow 0
$$

и длинную точную последовательность гомологий

$$
\cdots \rightarrow Y \stackrel{i_{*}}{\longrightarrow} H_{*}\left(K \otimes_{\phi} Y\right) \stackrel{p_{*}}{\longrightarrow} H_{*}\left(K \otimes_{\phi}^{1} Y\right) \stackrel{\phi_{*}}{\longrightarrow} Y \rightarrow \cdots
$$

ОПРЕДЕЛЕНИЕ 8. Элемент $y \in Y$ назовем разложимы.м, если он принадлежит образу гомоморфизма $\phi_{*}$, или, что то же самое, ядру гомоморфизма $i_{*}$. Фактормодуль модуля $Y$ по модулю неразложимых элементов называется модулем неразложимих әлементов.

Модуль неразложимых элементов модуля $Y$ обозначим $J Y$. Он будет изоморфен образу гомоморфизма $i_{*}$.

Пусть $X$ - градуированный комодуль над коалгеброй $K, \tau: X \rightarrow K \otimes X$. Элемент $x \in X$ назьвается примитивным м, если $\tau(x)=1 \otimes x$. Модуль примитивных элементов обозначим $P X$.

Взаимосвязь между примитивными элементами в $X$ и неразложимыми элементами в гомологиях ко- $B$-конструкции $Y=F(K, X)$ дает следуюшее

ПРЕДЛОЖЕНИЕ 24. Для градуированного комодуля $X$ над коалгеброй $K$ модуль неразлодсимых элементов $J Y_{*}$ модуля Ли $Y_{*}=H_{*}(F(K, X))$ над коалгеброй $K$ изоморфен модулю примитивных элементов $P X$.

Действительно, воспользуемся цепной эквивалентностью $X \simeq K \otimes_{\phi} Y \simeq$ $K \otimes_{\phi_{*}} Y_{*}$. Тогда имеют место изоморфизмы

$$
J Y_{*} \cong \operatorname{Im}\left\{i_{*}: Y_{*} \rightarrow H_{*}\left(K \otimes_{\phi_{*}} Y_{*}\right)\right\} \cong \operatorname{Im}\left\{Y_{*} \rightarrow X\right\} \cong \operatorname{Im}\left\{H_{*}(F(K, X)) \rightarrow X\right\}
$$

Образ последнего гомоморфизма совпадает с модулем примитивных элементов в $X$, что и завершает доказательство.

Для нахождения соотношений в градуированном модуле Ли $Y$ над коалгеброй $K$ нужно найти элементы $\sum x \otimes y \in K \otimes Y$, для которых $\sum \phi(x \otimes y)=0$. Конечно, некоторые из таких соотношений являются следствиями структуры $K$-модуля Ли в $Y$. А именно, если $x \otimes y \in K \otimes Y$, то элемент $d(x) \otimes y+\sum x^{\prime} \otimes \phi\left(x^{\prime \prime} \otimes y\right)$, где $\sum x^{\prime} \otimes x^{\prime \prime}=\nabla(x)$, порождает соотношение, являюшееся следствием структуры $K$-модуля Ли в $Y$. Профакторизуем $K \otimes Y$ по таким элементам и полученньй фактормодуль обозначим $K * Y$. Тогда для нахождения соотношений, которые не следуют просто из структуры $K$-модуля Ли, достаточно найти такие элементы в $K * Y$, которые переводятся в нуль отображением $\phi_{*}: K * Y \rightarrow Y$. 
Пусть $X$ - градуированный комодуль над коалгеброй $K, \tau: X \rightarrow K \otimes X$. Для нахождения соотношений в $K$-модуле Ли $Y_{*}=H_{*}(F(K, X))$ рассмотрим коммутативную диаграмму

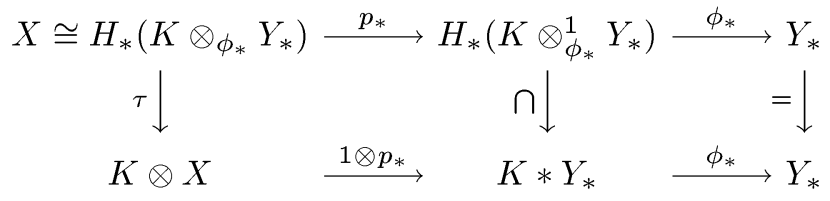

Из нее следует, что $\operatorname{Ker} \phi_{*}=\operatorname{Im}\left(1 \otimes p_{*}\right) \tau$, и, таким образом, искомые соотношения определяются элементами из образа отображения $X \stackrel{\tau}{\longrightarrow} K \otimes X \stackrel{1 \otimes p_{*}}{\longrightarrow} K * Y_{*}$. Поэтому для нахождения соотношений достаточно найти образ этого отображения.

Перейдем теперь к алгебрам Ли над операдой. Пусть $Y$ - градуированная алгебра Ли над операдой $\mathscr{E}, \phi: \overline{\mathscr{E}} Y \rightarrow Y$. Вложение $i: Y \rightarrow \overline{\mathscr{E}}_{\phi}(Y)$ индуцирует короткую точную последовательность

$$
0 \rightarrow Y \stackrel{i}{\rightarrow} \overline{\mathscr{E}}_{\phi}(Y) \stackrel{p}{\rightarrow} \overline{\mathscr{E}}_{\phi}^{1}(Y) \rightarrow 0
$$

и длинную точную последовательность гомологий

$$
\cdots \rightarrow Y \stackrel{i_{*}}{\longrightarrow} H_{*}\left(\overline{\mathscr{E}}_{\phi}(Y)\right) \stackrel{p_{*}}{\longrightarrow} H_{*}\left(\overline{\mathscr{E}}_{\phi}^{1}(Y)\right) \stackrel{\phi_{*}}{\longrightarrow} Y \rightarrow \cdots
$$

Элемент $y \in Y$ назовем разложсимым, если он принадлежит образу гомоморфизма $\phi_{*}$, или, что то же самое, ядру гомоморфизма $i_{*}$. Фактормодуль модуля $Y$ по модулю разложимых элементов называется модулем неразложимых әлементов и обозначается $J Y$. Модуль неразложимых элементов $J Y$ будет изоморфен образу гомоморфизма $i_{*}$.

Для градуированной коалгебры $X$ над операдой $\mathscr{E}$ модуль примитивньг элементов $P X$ определяется как образ гомоморфизма $p_{*}: H_{*}(F(\overline{\mathscr{E}}, X)) \rightarrow X$. Взаимосвязь между примитивными элементами в $X$ и неразложимыми элементами в гомологиях ко- $B$-конструкции $Y=F(\overline{\mathscr{E}}, X)$ дает следующее

ПРЕДЛОЖЕНИЕ 25. Для градуированной коалгебры $X$ над операдой $\mathscr{E}$ модуль неразложимых әлементов $J Y_{*}$ алгебры Ли $Y_{*}=H_{*}(F(\overline{\mathscr{E}}, X))$ над операдой Е изоморфен модулю примитивных әлементов $P X$.

Доказательство аналогично предыдушему.

Применяя это предложение к гомотопическим группам топологического пространства (точнее, к члену $E^{\infty}$ спектральной последовательности БоусфилдаКана), рассматриваемых как $E_{\infty}$-алгебра Ли, получаем следующую теорему.

ТЕОРема 2. Модуль неразложимых әлементов $J \pi_{*}(X)$ гомотопических зрупп односвязного топологического пространства $X$ изоморфен образу гомоморфизма Гуревича $\pi_{*}(X) \rightarrow H_{*}(X)$.

Доказательство следует из того, что $H_{*}\left(\bar{E}_{\infty \phi_{*}}\left(\pi_{*}(X)\right)\right) \cong H_{*}(X)$ (предложение 21$)$, и того, что гомоморфизм $i_{*}: \pi_{*}(X) \rightarrow H_{*}\left(\bar{E}_{\infty \phi_{*}}\left(\pi_{*}(X)\right)\right) \cong H_{*}(X)$ является гомоморфизмом Гуревича. 
Для нахождения соотношений в алгебре Ли над операдой поступаем так же, как и для модулей Ли над коалгеброй. Пусть $X$ - коалгебра над операдой $\mathscr{E}, Y=$ $F(\overline{\mathscr{E}}, X)$. Рассмотрим коммутативную диаграмму

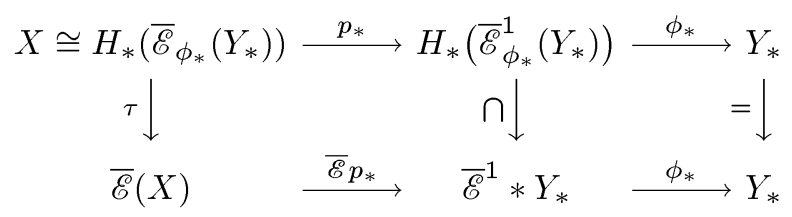

Соотношения в $Y_{*}$, не являюшиеся следствием структуры $\mathscr{E}$-алгебры Ли, определяются элементами из образа отображения $X \stackrel{\tau}{\longrightarrow} \overline{\mathscr{E}}(X) \stackrel{\mathscr{E} p_{*}}{\longrightarrow} \overline{\mathscr{E}}^{1} * Y_{*}$. Поэтому для нахождения соотношений достаточно найти образ этого отображения.

Применяя этот метод нахождения соотношений для гомотопических групп топологических пространств, получаем следуюшую теорему.

Теорема 3. Для гомотопических групп $\pi_{*}(X)$ односвязного топологического пространства $X$, рассматриваемых как $E_{\infty}$-алгебра Ли, все соотношения определяются әлементами из образа отображения

$$
H_{*}(X) \rightarrow \bar{E}_{\infty}\left(H_{*}(X)\right) \rightarrow \bar{E}_{\infty}^{1} * \pi_{*}(X),
$$

т.е. определяются структурой $E_{\infty}$-коалгебры на гомология $H_{*}(X)$ топологического пространства $X$.

В случае, если $X=S^{n}-n$-мерная сффера, получаем, что гомотопические группы $\pi_{*}\left(S^{n}\right)$, рассматриваемые как $E_{\infty}$-алгебра Ли, имеют одну $n$-мерную образующую и все соотношения следуют из структуры $E_{\infty}$-алгебры Ли.

\section{§ 12. Бимодули Хопфа и бимодули Ли}

В следуюшем параграфе мы рассмотрим вопрос о том, какая структура, помимо структуры $E_{\infty}$-алгебры Ли, возникает на гомотопических группах топологического пространства $X$ в случае, если топологическое пространство $X$ само является $E_{\infty}$-пространством, например бесконечнократньм пространством петель. Установим взаимосвязь между этими структурами, но сначала рассмотрим более простую ситуацию бимодулей.

Пусть $K$ - коалгебра, $A$ - алгебра, $T: A \otimes K \rightarrow K \otimes A$-перестановочное отображение, согласованное со структурами алгебры и коалгебры, т.е. коммутативны следуюшие диаграммы:

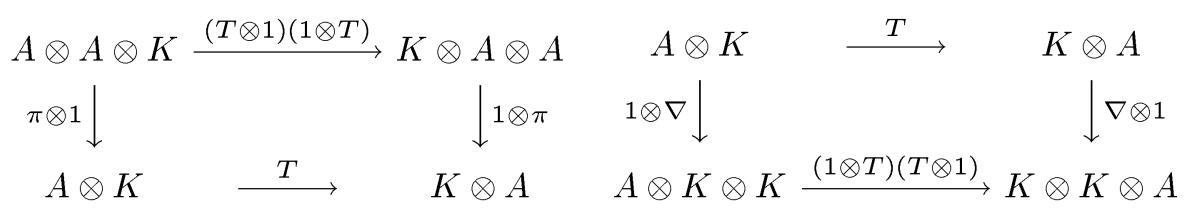

Модуль $X$ будем называть $(A, K)$-бимодулем, если на $X$ имеются структура $K$-комодуля $\tau: X \rightarrow K \otimes X$, структура $A$-модуля $\mu: A \otimes X \rightarrow X$, причем они согласованы, т.е. $\tau \circ \mu=(1 \otimes \mu)(T \otimes 1)(1 \otimes \tau)$. 
Последнее равенство означает, что если на $K \otimes X$ мы введем структуру $A$-модуля

$$
A \otimes K \otimes X \stackrel{T \otimes 1}{\longrightarrow} K \otimes A \otimes X \stackrel{1 \otimes \mu}{\longrightarrow} K \otimes X
$$

то отображение $\tau: X \rightarrow K \otimes X$ будет $A$-модульным отображением. Двойственным образом если на $A \otimes X$ ввести структуру $K$-комодуля

$$
A \otimes X \stackrel{1 \otimes \tau}{\longrightarrow} A \otimes K \otimes X \stackrel{T \otimes 1}{\longrightarrow} K \otimes A \otimes X
$$

отображение $\mu: A \otimes X \rightarrow X$ будет $K$-комодульным отображением.

В некоторых случаях указанное выше равенство выполняется только с точностью до гомотопии $h: A \otimes X \rightarrow K \otimes X, d(h)=\tau \circ \mu-(1 \otimes \mu)(T \otimes 1)(1 \otimes \tau)$, и тогда приходится вводить и использовать понятие $A_{\infty}-(A, K)$-бимодуля, в структуре которого участвуют не только гомотопия $h$, но и высшие гомотопии $h(i, j): A^{\otimes i} \otimes$ $X \rightarrow K^{\otimes j} \otimes X$. Однако в случае, если алгебра $A$ и коалгебра $K$ являются свободными, можно ограничиться только гомотопией $h$, потребовав от нее дополнительно выполнимость равенств

$$
\begin{aligned}
& h(\pi \otimes 1)=h(1 \otimes \mu)+(1 \otimes \mu)(T \otimes 1)(1 \otimes h), \\
& (\nabla \otimes 1) h=(1 \otimes \tau) h+(1 \otimes h)(T \otimes 1)(1 \otimes \tau) .
\end{aligned}
$$

ОПРЕДЕЛЕНИЕ 9. Дифференциальный градуированный модуль $X$ будем назы-

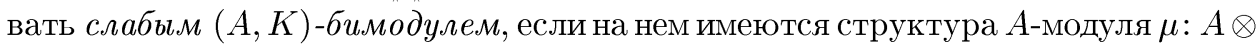
$X \rightarrow X$, структура $K$-комодуля $\tau: X \rightarrow K \otimes X$ и гомотопия $h: A \otimes X \rightarrow K \otimes X$, удовлетворяющая перечисленным выше условиям.

Аналогичным образом можно определить понятие $\left(A_{1}, A_{2}\right)$-бимодуля, где $A_{1}$, $A_{2}$ - алгебры с согласованными структурами алгебр, и понятие $\left(K_{1}, K_{2}\right)$-бикомодуля, где $K_{1}, K_{2}$ - коалгебры с согласованными структурами коалгебр.

Если $X-(A, K)$-бимодуль, то ко- $B$-конструкция $F(K, X)$ является $F K$-модулем и $A$-модулем. Перестановочное отображение $T: A \otimes K \rightarrow K \otimes A$ индуцирует перестановочное отображение $A \otimes F K \rightarrow F K \otimes A$, относительно которого эти структуры согласованы, т.е. $F(K, X)$ является $(A, F K)$-бимодулем. Двойственным образом $B$-конструкция $B(A, X)$ является $(B A, K)$-бикомодулем.

ОПРЕДЕЛЕНИЕ 10. Пусть, как и ранее, $A$ - алгебра, $K$ - коалгебра. Дифференциальньй градуированный модуль $Y$ будем называть $(A, K)$-бимодулем Ли, если $Y$ является $A$-модулем, $K$-модулем Ли и эти структуры согласованы, т.е. имеет место равенство $\mu(1 \otimes \phi)=\phi(1 \otimes \mu)(T \otimes 1): A \otimes K \otimes Y \rightarrow Y$.

Определим также понятие слабого $(A, K)$-бимодуля Ли.

ОПРЕДЕЛЕНИЕ 11. Дифференциальный градуированный модуль $Y$ будем называть слабы.м $(A, K)$-бимодулем Ли, если на нем имеются структуры $A$-модуля $\mu: A \otimes Y \rightarrow Y$, структура $K$-модуля Ли $\phi: K \otimes Y \rightarrow Y$ и отображение $c: A \otimes$ $K \otimes Y \rightarrow Y$, удовлетворяющее условиям

$$
\begin{gathered}
d(c)=c(1 \otimes 1 \otimes \phi)(1 \otimes \nabla \otimes 1)-\phi(1 \otimes c)(T \otimes 1 \otimes 1)(1 \otimes \nabla \otimes 1), \\
c(\pi \otimes 1 \otimes 1)=c(1 \otimes 1 \otimes c)(1 \otimes T \otimes 1 \otimes 1)(1 \otimes 1 \otimes \nabla \otimes 1) \\
c(x \otimes 1 \otimes y)=\mu(x \otimes y) .
\end{gathered}
$$


Примеры $(A, K)$-бимодулей Ли дают рассмотренные выше ко- $B$-конструкции $F(K, X)$ над $(A, K)$-бимодулем $X$. Причем если $X$ - слабый $(A, K)$-бимодуль, то $Y=F(K, X)$ является просто бимодулем Ли. Обратно, если $Y$ - слабый $(A, K)$-бимодуль Ли, то $X=K \otimes_{\phi} Y$ будет просто $(A, K)$-бимодулем.

Естественные преобразования

$$
\alpha(X): X \rightarrow K \otimes_{\phi} F(K, X), \quad \beta(Y): F\left(K, K \otimes_{\phi} Y\right) \rightarrow Y
$$

сохраняют бимодульную структуру и являются цепными эквивалентностями.

Рассмотрим теперь вопрос о том, какая структура возникает на гомологиях $(A, K)$-бимодуля Ли $Y$. В этом случае на $Y$ имеются согласованные структура $K$-комодуля Ли $\phi: K \otimes Y \rightarrow Y$ и структура $A$-модуля $\mu: A \otimes Y \rightarrow Y$. На гомологиях $Y_{*}=H_{*}(Y)$ будет структура $K$-модуля Ли $\phi_{*}: K \otimes Y_{*} \rightarrow Y_{*}$ и структура $A_{\infty}$ - $A$-модуля $\mu_{*}^{n}: A^{\otimes n+1} \otimes Y_{*} \rightarrow Y_{*}, n \geqslant 0$. Поскольку мы рассматриваем случай, когда алгебра $A$ является свободной, то структура $A_{\infty}-A$-модуля сводится к структуре $A$-модуля $\mu_{*}: A \otimes Y_{*} \rightarrow Y_{*}$. Однако даже в этом случае структуры $\phi_{*}$ и $\mu_{*}$ не являются согласованными и не дают структуру $(A, K)$-бимодуля Ли на $Y_{*}$, а только структуру слабого бимодуля Ли.

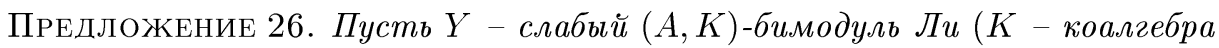
конечного типа). Тогда на гомологиях $Y_{*}=H_{*}(Y)$ имеется структура слабого $(A, K)$-бимодуля Ли и чепная әквивалентность $K \otimes_{\phi} Y \simeq K \otimes_{\phi_{*}} Y_{*}$ $(A, K)-$ бимодулей.

Действительно, структура слабого $(A, K)$-бимодуля Ли на $Y$ определяет структуру $A$-модуля на $K \otimes_{\phi} Y$. Следовательно, на $K \otimes_{\phi_{*}} Y_{*}$ также имеется структура $A$-модуля $A \otimes K \otimes_{\phi_{*}} Y_{*} \rightarrow K \otimes_{\phi_{*}} Y_{*}$ (алгебра $A$ свободна как градуированная алгебра). Поскольку приведенная вьше цепная эквивалентность скрещенных тензорных произведений сохраняет $K$-комодульную структуру, то эта $A$-модульная структура согласована с $K$-комодульной структурой. Следовательно, на $K \otimes_{\phi_{*}} Y_{*}$ имеется структура $(A, K)$-бимодуля. Заметим, что задание $K$-комодульного отображения $A \otimes K \otimes_{\phi_{*}} Y_{*} \rightarrow K \otimes_{\phi_{*}} Y_{*}$ равносильно заданию отображения с ${ }_{*}: A \otimes$ $K \otimes_{\phi_{*}} Y_{*} \rightarrow Y_{*}$, удовлетворяющего соотношению $d\left(\mathrm{c}_{*}\right)=\mathrm{c}_{*} \cup \phi_{*}-\phi_{*} \cup \mathrm{c}_{*}$. Для того чтобы исходное отображение было $A$-модульным, нужно, чтобы выполнялось соотношение $\mathrm{c}_{*}(\pi \otimes 1 \otimes 1)=\nu_{*} \cup \mathrm{c}_{*}$. Ограничение $\mathrm{c}_{*}$ на $A \otimes Y_{*} \subset A \otimes K \otimes Y_{*}$ дает структуру $A$-модуля на $Y_{*}$.

Как и раньше, в случае, если коалгебра $K$ не является коалгеброй конечного типа, нужно рассматривать фильтрацию в $Y$, и тогда для пополнения $\widehat{Y}_{*}$ будет иметь место аналогичное предложение.

\section{$\S 13 . E_{\infty}$-алгебры Хопфа и $E_{\infty}$-биалгебры Ли}

Понятия бимодуля Хопфа и бимодуля Ли, рассмотренные в предыдущем параграфе, можно распространить на случай алгебр и коалгебр над операдами. Для нас будет достаточным рассмотрение случая, когда алгебры и коалгебры берутся над операдой $E_{\infty}$.

Пусть теперь, как и раньше, $E_{\infty}$ и $\bar{E}_{\infty}$ обозначают соответственно монаду и комонаду в категории цепных комплексов, соответствующую операде $E_{\infty}$. 
ПРЕДЛОЖЕНИЕ 27. Для операды $E_{\infty}$ имеет место перестановочное преобразование $T: E_{\infty} \circ \bar{E}_{\infty} \rightarrow \bar{E}_{\infty} \circ E_{\infty}$, для которого выполняются равенства

$$
T \circ \gamma \bar{E}_{\infty}=\bar{E}_{\infty} \gamma \circ T E_{\infty} \circ E_{\infty} T, \quad \bar{\gamma} E_{\infty} \circ T=\bar{E}_{\infty} T \circ T \bar{E}_{\infty} \circ E_{\infty} \bar{\gamma} .
$$

Действительно, имеет место отображение операд $\nabla: E_{\infty} \rightarrow E_{\infty} \otimes E_{\infty}$, представляющее собой семейство отображений $\nabla(j): E_{\infty}(j) \rightarrow E_{\infty}(j) \otimes E_{\infty}(j)$, задающее на $E_{\infty}$ структуру операды Хопфа. Рассмотрим итерацию этого отображения $\nabla(j, i): E_{\infty}(j) \rightarrow E_{\infty}(j)^{\otimes i}, \nabla(j, 2)=\nabla(j)$. Это отображение, конечно, не коммутирует с перестановками сомножителей в $E_{\infty}(j)^{\otimes i}$, однако в силу ацикличности и $\Sigma$-свободности операды $E_{\infty}$ его можно продолжить до отображения $\nabla(j, i): E_{\infty}(i) \otimes E_{\infty}(j) \rightarrow E_{\infty}(j)^{\otimes i}$, для которого будет вьполняться равенство $\nabla(j, i)(x \sigma \otimes y)=\nabla(j, i)(x \otimes y) \sigma$, где $\sigma \in \Sigma_{i}$.

Перепишем это отображение в виде $\nabla(j, i): E_{\infty}(j) \rightarrow \bar{E}_{\infty}(i) \otimes E_{\infty}(j)^{\otimes i}$. В силу свободности операды $E_{\infty}$ его можно выбрать согласованным с операдной структурой в $E_{\infty}$. Переходя к двойственному отображению, получим отображение $\bar{\nabla}(j, i)$ : $E_{\infty}(i) \otimes \bar{E}_{\infty}(j)^{\otimes i} \rightarrow \bar{E}_{\infty}(j)$.

Определим теперь отображение $T(j, i): E_{\infty}(j) \otimes \bar{E}_{\infty}(i)^{\otimes j} \rightarrow E_{\infty}(i) \otimes \bar{E}_{\infty}(j)^{\otimes i}$ как композищию

$$
\begin{aligned}
E_{\infty}(j) \otimes \bar{E}_{\infty} & (i)^{\otimes j} \stackrel{\stackrel{\nabla(j) \otimes 1^{\otimes j}}{\longrightarrow} E_{\infty}(j) \otimes E_{\infty}(j) \otimes \bar{E}_{\infty}(i)^{\otimes j}}{\rightarrow} E_{\infty}(j) \otimes \bar{E}_{\infty}(i)^{\otimes j} \otimes E_{\infty}(j) \stackrel{\bar{\nabla}(j, i) \otimes 1}{\longrightarrow} \bar{E}_{\infty}(i) \otimes E_{\infty}(j) \\
& \stackrel{1 \otimes \nabla(j, i)}{\longrightarrow} \bar{E}_{\infty}(i) \otimes \bar{E}_{\infty}(i) \otimes E_{\infty}(j)^{\otimes i} \stackrel{\bar{\nabla}(i) \otimes 1^{\otimes i}}{\longrightarrow} \bar{E}_{\infty}(j) \otimes E_{\infty}(i)^{\otimes j} .
\end{aligned}
$$

Семейство отображений $T(j, i)$ определяет искомое перестановочное отображение $T: E_{\infty} \circ \bar{E}_{\infty} \rightarrow \bar{E}_{\infty} \circ E_{\infty}$. Непосредственные вычисления показывают коммутативность требуемых диаграмм.

В случае, если $X$ является $E_{\infty}$-алгеброй, перестановочное отображение $T: E_{\infty}$ ० $\bar{E}_{\infty} \rightarrow \bar{E}_{\infty} \circ E_{\infty}$ позволяет определить на $\bar{E}_{\infty} X$ структуру $E_{\infty}$-алгебры как композищию $E_{\infty} \circ \bar{E}_{\infty}(X) \stackrel{T}{\longrightarrow} \bar{E}_{\infty} \circ E_{\infty}(X) \stackrel{\bar{E}_{\infty} \mu}{\longrightarrow} \bar{E}_{\infty}(X)$.

Аналогично, если $X$ является $E_{\infty}$-коалгеброй, то перестановочное отображение позволяет определить на $E_{\infty}(X)$ структуру $E_{\infty}$-коалгебры как композищию $E_{\infty}(X) \stackrel{E_{\infty} \tau}{\longrightarrow} E_{\infty} \circ \bar{E}_{\infty}(X) \stackrel{T}{\longrightarrow} \bar{E}_{\infty} \circ E_{\infty}(X)$

ОПРЕДЕЛЕНИЕ 12. Будем говорить, что дифференииальный градуированный модуль $X$ является $E_{\infty}$-алгеброй Хопфа, если на нем заданы структуры $E_{\infty}$-алгебры $\mu: E_{\infty}(X) \rightarrow X$ и $E_{\infty}$-коалгебры $\tau: X \rightarrow E_{\infty}(X)$, которые согласованы в том смысле, что отображение $\tau$ является отображением $E_{\infty}$-алгебр, или, что то же самое, отображение $\mu$ является отображением $E_{\infty}$-коалгебр.

В некоторых случаях указанная вьше согласованность выполняется только с точностью до гомотопии $h: E_{\infty}(X) \rightarrow \bar{E}_{\infty}(X), d(h)=\tau \circ \mu-\bar{E}_{\infty}(\mu)(T X) E_{\infty}(\tau)$. Учитывая, что операда $E_{\infty}$-свободна, можно ограничиться только гомотопией $h$, потребовав от нее дополнительно выполнимость равенств

$$
\begin{aligned}
& h \circ \gamma(X)=h \circ E_{\infty}(\mu)+\bar{E}_{\infty}(\mu)(T X) \circ E_{\infty}(h), \\
& \bar{\gamma}(X) \circ h=\bar{E}_{\infty}(\tau) \circ h+\bar{E}_{\infty}(h) \circ(T X) \circ E_{\infty}(\tau) .
\end{aligned}
$$


ОПРЕДЕЛЕниЕ 13. Дифференциальный градуированный модуль $X$ будем называть слабой $E_{\infty}$-алгеброй Хопфа, если на нем имеются структура $E_{\infty}$-алгебры $\mu: E_{\infty}(X) \rightarrow X$, структура $E_{\infty}$-коалгебры $\tau: X \rightarrow \bar{E}_{\infty}(X)$ и гомотопия $h: E_{\infty}(X) \rightarrow \bar{E}_{\infty}(X)$, удовлетворяюшая перечисленным вьше условиям.

Пусть $X$ является $E_{\infty}$-алгеброй Хопфа. Рассмотрим ко- $B$-конструкцию $F\left(E_{\infty}\right.$, $X)$. Тогда поскольку эта ко- $B$-конструкция является реализацией косимплициального объекта в категории $E_{\infty}$-алгебр, то она является $E_{\infty}$-алгеброй. Кроме этого, как мы знаем, $F\left(E_{\infty}, X\right)$ является $E_{\infty}$-алгеброй Ли.

ОПРЕДЕЛЕниЕ 14. Дифференциальньй градуированный модуль $Y$ будем называть $E_{\infty}$-биалzеброй Лu, если на нем заданы согласованные структуры $E_{\infty}$-алгебры и $E_{\infty}$-алгебры Ли.

Определим также понятие слабой $E_{\infty}$-биалгебры Ли.

ОПРЕДЕЛЕниЕ 15. Дифференциальный градуированный модуль $Y$ будем называть слабой $E_{\infty}$-биалгеброй Ли, если на нем имеются структура $E_{\infty}$-алгебры $\mu: E_{\infty}(Y) \rightarrow Y$, структура $E_{\infty}$-алгебры Ли $\phi: \bar{E}_{\infty}(Y) \rightarrow Y$ и отображение $c: E_{\infty} \bar{E}_{\infty}(Y) \rightarrow Y$, удовлетворяющее условиям

$$
\begin{gathered}
d(c)=c \circ E_{\infty} \bar{E}_{\infty}(\phi) \circ E_{\infty} \bar{\gamma}(Y)-\phi \circ \bar{E}_{\infty}(c) \circ\left(T \bar{E}_{\infty}(Y)\right) \circ E_{\infty} \bar{\gamma}(Y), \\
c \circ \gamma \bar{E}_{\infty}(Y)=c \circ E_{\infty} \bar{E}_{\infty}(c) \circ\left(E_{\infty} T \bar{E}_{\infty}(Y)\right) \circ E_{\infty} E_{\infty} \bar{\gamma}(Y), \\
c(x \times 1 \times y)=\mu(x \times y) .
\end{gathered}
$$

Примеры $E_{\infty}$-биалгебр Ли дают рассмотренные выше ко- $B$-конструкции $F\left(\bar{E}_{\infty}, X\right)$ над $E_{\infty}$-алгеброй Хопфа $X$. Причем если $X$ - слабая $E_{\infty}$-алгебра Хопфа, то $Y=F\left(\bar{E}_{\infty}, X\right)$ является просто биалгеброй Ли. Обратно, если $Y$ - слабая $E_{\infty}$-биалгебра Ли, то $X=\bar{E}_{\infty \phi}(Y)$ будет просто $E_{\infty}$-биалгеброй Хопфа.

Так же, как и в случае бимодулей Ли, показывается, что имеет место следующее

ПРЕДЛОЖЕНИЕ 28. Пусть $Y-E_{\infty}$-слабая биалгебра Ли. Тогда на гомологиях $Y_{*}=H_{*}(Y)$ имеется структура слабой $E_{\infty}$-биалгебры Ли и чепная эквивалентность $E_{\infty}$-алгебр Хопфа $\bar{E}_{\infty \phi}(Y) \simeq \bar{E}_{\infty \phi_{*}}\left(Y_{*}\right)$.

Применяя это предложение к гомотопическим группам, получаем следующую теорему.

ТеОрема 4. Если односвязное топологическое пространство $X$ является $E_{\infty}$-пространством, то на гомотопических группах $\pi_{*}(X)$ (точнее, на члене $E^{\infty}$ спектральной последовательности Боусфилда-Кана) имеется структура слабой $E_{\infty}$-биалгебры Ли. В частности, такая структура имеется на стабильных гомотопических группах топологического пространства. 


\section{Список литературы}

1. Смирнов B. A. $E_{\infty}$-структуры на гомотопических группах // Матем. заметки. 1997. T. 61 . № 1. C. $152-156$.

2. Brown E. H. Twisted tensor products // Ann. of Math. 1959. V. 69. P. 223-246.

3. Gugenheim V.K.A.M., Lambe L. A., Stasheff J.D. Perturbation Theory in Differential Homological Algebra // Ill. J. Math. 1991. V. 35. № 3. P. 357-373.

4. Смирнов В. А. Гомологии скрещенных тензорных произведений // ДАН СССР. 1975. T. 222. № 5. C. 1041-1044.

5. Quillen D. Rational homotopy theory // Ann. of Math. 1969. V. 90. № 2. P. 205-295.

6. Серр Ж.-П. Алгебры Ли и группы Ли. М.: Мир, 1969.

7. May J. P. The Geometry of Iterated Loop Spaces // Lect. Notes in Math. 1972. V. 271.

8. Смирнов B. A. О коцепном комплексе топологического пространства // Матем. сб. 1981. T. 115. № 1. C. 146-158.

9. Stasheff J. D. Homotopy associativity of H-spaces // Trans. A.M.S. 1963. V. 108. № 2 . P. 275-312.

10. Кадеишвили Т. В. К теории гомологий расслоенных пространств // УМН. 1980. Т. 35. C. $183-188$.

11. Смирнов В. А. Гомологии расслоенных пространств // УМН. 1980. Т. 35. С. 227-230.

12. Смирнов В. А. Гомотопическая теория коалгебр // Изв. АН СССР. Сер. метем. 1985. T. 49. №6. C. 1302-1321.

13. Смирнов B. А. Вторичные операции в гомологиях операды $E$ // Изв. РАН. Сер. матем. 1992. T. 56. № 2. C. 449-468.

14. Смирнов $B . A$. Функциональные гомологические операции и слабый гомотопический тип // Матем. заметки. 1989. Т. 45. № 5. С. 76-86.

15. Смирнов B. A. Гомологии $B$-конструкций и ко- $B$-конструкций // Изв. РАН. Сер. матем. 1994. Т. 58. № 4. С. 80-96.

16. Bousfield A.K., Kan D. M. The homotopy spectral sequence of a space with coefficients in a ring // Topology. 1972. V. 11. P. 79-106.

17. Massey W.S. Products in exact couples // Ann. of Math. 1954. V. 59. P. 558-569.

Поступило в редакцию

12.III. 1996 